\title{
Dysfunctional polycomb transcriptional repression contributes to lamin A/C-dependent muscular dystrophy
}

\author{
Andrea Bianchi, ${ }^{1,2}$ Chiara Mozzetta, ${ }^{2}$ Gloria Pegoli, ${ }^{3}$ Federica Lucini, ${ }^{1}$ Sara Valsoni, ${ }^{1,3}$ Valentina Rosti, ${ }^{4}$ Cristiano Petrini, ${ }^{5}$ \\ Alice Cortesi, ${ }^{1}$ Francesco Gregoretti, ${ }^{6}$ Laura Antonelli, ${ }^{6}$ Gennaro Oliva, ${ }^{6}$ Marco De Bardi, ${ }^{3}$ Roberto Rizzi, ${ }^{1,4}$ Beatrice Bodega, \\ Diego Pasini, ${ }^{7,8}$ Francesco Ferrari, ${ }^{5,9}$ Claudia Bearzi, ${ }^{1,10}$ and Chiara Lanzuolo ${ }^{3,4}$ \\ "Istituto Nazionale di Genetica Molecolare “Romeo ed Enrica Invernizzi," Milan, Italy. ${ }^{2}$ Institute of Cell Biology and Neurobiology, National Research Council (CNR), Rome, Italy. ${ }^{3}$ Santa Lucia Foundation, \\ Scientific Institute for Research, Hospitalization and Healthcare (IRCCS), Rome, Italy. ${ }^{4}$ Institute of Biomedical Technologies, CNR, Milan, Italy. ${ }^{5}$ IFOM, the FIRC Institute of Molecular Oncology, Milan, Italy. \\ ${ }^{6}$ Institute for High Performance Computing and Networking, CNR, Naples, Italy. IEO European Institute of Oncology IRCCS, Department of Experimental Oncology, Milan, Italy. ${ }^{8}$ Department of Health \\ Sciences, University of Milan, Milan, Italy. ${ }^{9}$ Institute of Molecular Cenetics “Luigi Luca Cavalli-Sforza," CNR, Pavia, Italy. ${ }^{10}$ Institute of Biochemistry and Cell Biology, CNR, Rome, Italy.
}

\begin{abstract}
Lamin A is a component of the inner nuclear membrane that, together with epigenetic factors, organizes the genome in higher order structures required for transcriptional control. Mutations in the lamin A/C gene cause several diseases belonging to the class of laminopathies, including muscular dystrophies. Nevertheless, molecular mechanisms involved in the pathogenesis of lamin A-dependent dystrophies are still largely unknown. The polycomb group (PcG) of proteins are epigenetic repressors and lamin A interactors, primarily involved in the maintenance of cell identity. Using a murine model of Emery-Dreifuss muscular dystrophy (EDMD), we show here that lamin A loss deregulated PcG positioning in muscle satellite stem cells, leading to derepression of non-muscle-specific genes and p16 ${ }^{\mid \mathrm{NK} 4 \mathrm{a}}$, a senescence driver encoded in the Cdkn2a locus. This aberrant transcriptional program caused impairment in self-renewal, loss of cell identity, and premature exhaustion of the quiescent satellite cell pool. Genetic ablation of the Cdkn2a locus restored muscle stem cell properties in lamin A/C-null dystrophic mice. Our findings establish a direct link between lamin A and PcG epigenetic silencing and indicate that lamin A-dependent muscular dystrophy can be ascribed to intrinsic epigenetic dysfunctions of muscle stem cells.
\end{abstract}

\section{Introduction}

The nuclear lamina (NL) is located in the inner part of the nuclear membrane and is composed of a complex network of type $\mathrm{V}$ filament proteins, the lamins $(1,2)$. In vertebrates, lamin proteins are divided into A and B types, based on sequence homologies. A growing body of evidence suggests that lamins are directly involved in the functional control of the genome, by organizing its $3 \mathrm{D}$ positioning in the nuclear space through the association with transcriptionally repressed large genomic regions, called lamina-associated domains (LADs) (3). The crucial function of lamins is attested by an entire class of genetic diseases, called laminopathies, in which specific components of the NL are altered (4). In particular, the study of lamin A/C is gaining an increasing interest for 3 reasons: (a) lamin A/C plays an undisputed role in several cellular processes from mechanotransduction to cell differentiation; (b) lamin $\mathrm{A} / \mathrm{C}$ has a peculiar intranuclear distribution, being present in the nucleoplasm as well as in

Authorship note: BA and MC contributed equally to this work. Conflict of interest: The authors have declared that no conflict of interest exists. Copyright: ( 2020 , American Society for Clinical Investigation. Submitted: February 14, 2019; Accepted: January 22, 2020; Published: April 6, 2020. Reference information: / Clin Invest. 2020;130(5):2408-2421. https://doi.org/10.1172/JCl128161. the nuclear periphery (5); (c) lamin A/C interacts with several epigenetic factors, exerting a functional control over transcriptional regulation $(3,6)$. One of the most studied lamin A/Cdependent cellular process is myogenesis because mutations in the LMNA gene lead to muscular dystrophies, as in the case of Emery Dreifuss muscular dystrophy (EDMD) (7). However, epigenetic mechanisms involved in lamin-dependent dystrophy are still largely unknown. The polycomb group $(\mathrm{PcG})$ of proteins are epigenetic repressors originally discovered for their central roles in development and cell differentiation (8) and were recently described as functional partners of lamin A/C (9-14). In recent years evidence has demonstrated that PcG proteins are involved in the regulation of adult stem cells $(15,16)$, safeguarding cell identity and preventing cell fate transition. In multipotent stem cells, PcG proteins ensure the correct balance between selfrenewal and lineage-specific differentiation, promptly responding to environmental changes. At the molecular level this is achieved through PcG binding at bivalent domains, genomic regions containing active and repressive epigenetic signatures, and a poised RNA polymerase II (17). This epigenetic condition allows a rapid transition from one transcriptional state to another, ensuring the correct expression of unique and specific cell lineage genes. Defects in these fine-tuned mechanisms lead to lack of cell identity (18) or pathological reprogramming (19). 
Given their key role in regulating stem cell fate decisions and tissue homeostasis, it is conceivable that PcG dysfunctions contribute to lamin-dependent, tissue-specific human diseases. Here, we examined how the absence of lamin A/C impacts muscle stem (satellite) cells (MuSCs) in vivo, and the role of PcG proteins in lamin muscular dystrophy. We found that MuSCs lacking lamin A/C redistribute PcG-dependent histone marks, leading to transcriptional upregulation of crucial PcG-target genes, such as non-muscle-related genes. This leads to lack of MuSC identity and senescence, determining a premature exhaustion of the MuSC niche. Genetic ablation of the PcG-regulated Cdkn2a locus in lamin-dystrophic mice restores MuSCs defects.

\section{Results}

Lamin $A$ is required to preserve the quiescent MuSC pool. We analyzed the MuSC niche composition in the severely dystrophic Lmna $\Delta 8^{-11^{-/-}}$mice (homozygous, hom), together with their unaffected littermates, wild-type (WT, Lmna $\Delta 8-11^{+/+}$) or heterozygous (het, Lmna $\Delta 8^{-11^{+/-}}$), during dystrophy progression at 10,14,16, and 19 days after birth. In early stages of postnatal growth (day 10 and d14) no differences were found in the relative amounts of quiescent stem cells (QSCs; PAX7 ${ }^{+} \mathrm{MYOD}^{-}$) and activated stem cells (ASCs; $\mathrm{PAX}^{+} \mathrm{MYOD}^{+}$) (Figure 1, A and B, and Supplemental Figure 1A; supplemental material available online with this article; https:// doi.org/10.1172/JCI128161DS1) among MuSCs with distinct genotypes. Conversely, starting from d16, an imbalance of MuSCs becomes evident in Lmna $\Delta 8^{-11^{-/-}}$muscles, with a decreased proportion of QSCs compared with ASCs, mirroring a decline in myofiber cross-sectional area (CSA) (Figure 1C). Of note, the overall amount of $\mathrm{PAX}^{+}$MuSCs was not significantly altered across the different genotypes (Supplemental Figure 1B) and Ki67 staining

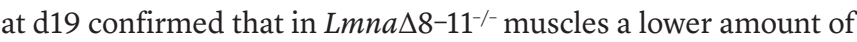
QSCs $\left(\mathrm{PAX7}^{+} \mathrm{Ki67}\right)$ is present (Supplemental Figure $1 \mathrm{C}$ ). These findings suggested that Lmna $\Delta 8-11^{-/-}$MuSCs may be deficient in self-renewal capacity. To test this hypothesis, we isolated single myofibers at d19 and cultured them for 96 hours, monitoring their ability to give rise to self-renewing $\mathrm{PAX}^{+} \mathrm{MYOD}^{-}$, activated $\mathrm{PAX}^{+} \mathrm{MYOD}^{+}$, and differentiating $\mathrm{PAX}^{-} \mathrm{MYOD}^{+}$cells (Figure 1, D and $\mathrm{E})$. In fibers isolated from $L m n a \Delta 8^{-11^{-/-}}$muscles, we observed a decrease in the number of self-renewing $\mathrm{PAX}^{+} \mathrm{MYOD}^{-}$cells compared with WT, paralleled by a diminished number of differentiating cells ( $\mathrm{PAX7}^{-} \mathrm{MYOD}^{+}$) and an increased number of activated satellite cells. Immunostaining with the myogenic marker MYOG, which is involved in later stages of differentiation, highlighted a lower number of $\mathrm{MYOG}^{+} \mathrm{PAX7}^{-}$cells in Lmna $8^{-11^{-/-}}$(Figure 1, F and $G$ ) accompanied by proliferation defects ex vivo (Supplemental Figure 1D). These findings suggest a defect in muscle differentiation, as described in Cohen et al. (20), and a previously unreported self-renewal impairment. Interestingly, the healthy heterozygous Lmna $\Delta 8-11^{+/-}$mice, although not developing muscular dystrophy (21), presented an intermediate self-renewal phenotype between

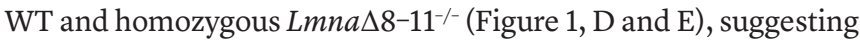
that proper lamin A levels are important for MuSC homeostasis to preserve their self-renewal capacity. To further address this, we repeatedly injured muscle in adult heterozygous $\operatorname{Lm} n a \Delta 8^{-11^{+/-}}$ mice, which show less lamin A at the mRNA and protein level (Supplemental Figure 2, A and B; see complete unedited blots in the supplemental material). Analysis of MuSC populations revealed

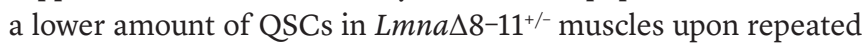
injuries (Figure 2, A and B) and a decline in $\mathrm{Pax}^{+}$cells (Figure 2C), suggesting that lamin A affects MuSC self-renewal in a dosedependent manner.

Lmna $48^{-11^{-/}}$dystrophic MuSCs display chromatin redistribution of PcG-dependent signature. Our recent results showed a lamin A/C-PcG crosstalk during in vitro myogenesis (10). We thus wondered if the altered MuSC balance observed in Lmna $\Delta 8^{-11^{-1-}}$ muscles might be ascribed to aberrant PcG functions. We first performed immunostaining of Ezh2, the catalytic subunit of polycomb repressive complex 2 (PRC2) (Supplemental Figure 3A) in d19 MuSCs. We fixed MuSCs before FACS isolation to preserve the nuclear architecture of lamin A-deficient cells (see Methods). We found a general intranuclear diffusion of Ezh2 in Lmna $\Delta 8^{-11^{-1-}}$ MuSCs, ascertained by measuring PcG body parameters (22) (Supplemental Figure 3, A-C). We also measured Ezh2 expression both in MuSCs and whole muscles (Supplemental Figure 3D) and we analyzed Ezh2 protein levels in whole muscles (Supplemental Figure 3, E and F; see complete unedited blots in the supplemental

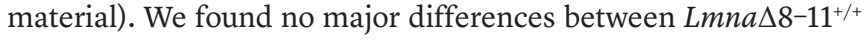
and Lmna $\Delta 8-11^{-/-}$mice. To further analyze the Ezh2 intranuclear distribution in QSCs, we performed triple immunostaining on muscle cryosections (Supplemental Figure 3, G and H). Ezh2 levels, assessed measuring fluorescence intensity, were simi-

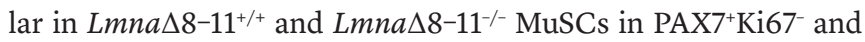
$\mathrm{PAX}^{+} \mathrm{Ki}^{+} 7^{+}$cells. Because Ezh2 is hardly detectable in adult quiescent satellite cells (23-25), this result suggests that during postnatal growth developmental signals might instead contribute to maintaining Ezh2 expression in nonproliferating MuSCs.

On the other hand, evaluation of the number of PcG bodies on the same sections highlighted a decrease in the number of Ezh2 bodies in the mutant (Supplemental Figure 3, I and J), leading us to conclude that the absence of lamin A/C does not affect Ezh2 protein levels but influences its nuclear distribution. To gain further insight into possible PcG-dependent transcriptional defects, we performed RNA sequencing (RNA-seq) on freshly isolated MuSCs at d19, finding 1424 upregulated genes and 1842 downregulated genes in the Lmna $\Delta 8-11^{-/-}$MuSCs compared with WT (Supplemental Figure 4A). Interestingly, performing a gene set enrichment analysis (GSEA) based on differential expression generated after conditional ablation of Ezh2 in MuSCs (24) and Lmna $\Delta 8^{-11^{-/-}}$ upregulated genes, we found a significant association between the 2 data sets, suggesting that lamin A absence impairs Ezh2 function (Supplemental Figure 4B). We also followed the deposition of the Ezh2-dependent H3K27me3 histone mark in Lmna $\Delta$ 8-11 mice by quantitative spike-in ChIP-seq (26) (see Supplemental Methods and Supplemental Figure 4, C and D). Integrative analysis of RNA-seq and ChIP-seq revealed that upregulated genes in the Lmna $\Delta 8-11^{-/-}$condition are significantly enriched for H3K27me3 targets (identified in the WT condition) (Figure 3A). Indeed, analysis of $\mathrm{H} 3 \mathrm{~K} 27 \mathrm{me} 3$ distribution around the transcription start sites (TSSs) and along the body of genes indicated a decrease in this repressive mark in Lmna $\Delta 8^{-11^{-/-}}$MuSCs compared with WT (Figure 3, B and C), which was not accompanied by a statistically significant decrease in global H3K27me3 levels in MuSCs (Supplemental Figure 5, A and B; see complete unedited blots in the 
A

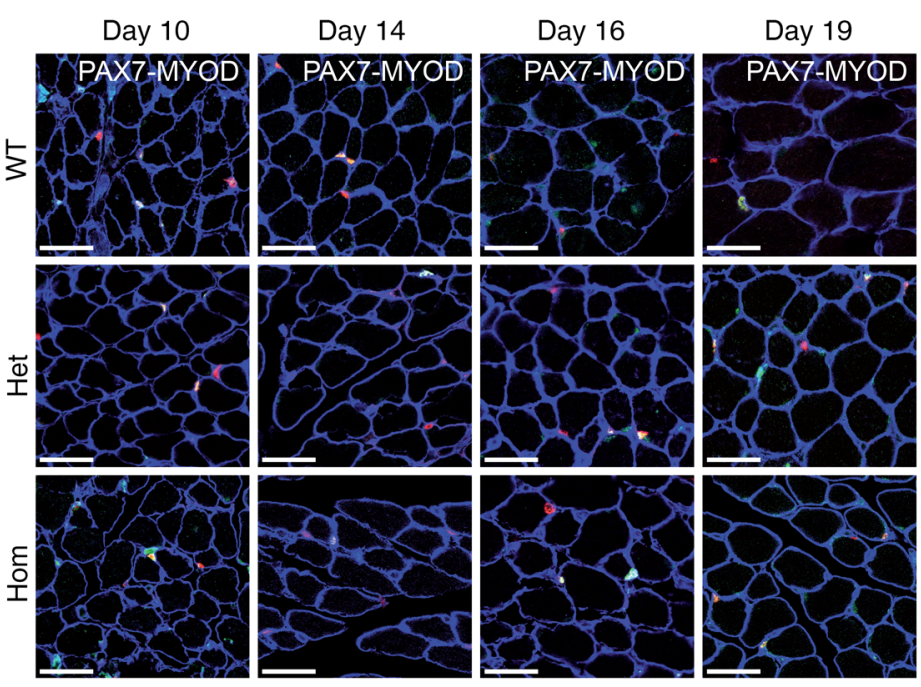

$\bullet:$ QSCs (PAX7 $\left.7^{+} \mathrm{MYOD}^{-}\right) \quad \bullet:$ ASCs $\left(\mathrm{PAX7}^{+} / \mathrm{MYOD}^{+}\right)$
B $\square \mathrm{MYOD}^{+}(\mathrm{ASCs}) \cdot \mathrm{MYOD}^{-}$(QSCs)

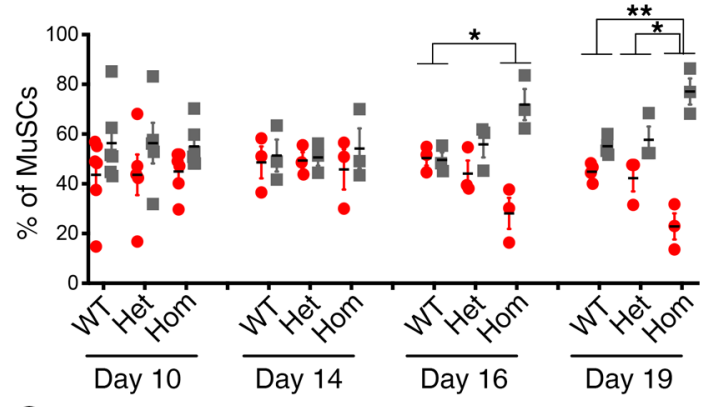

C

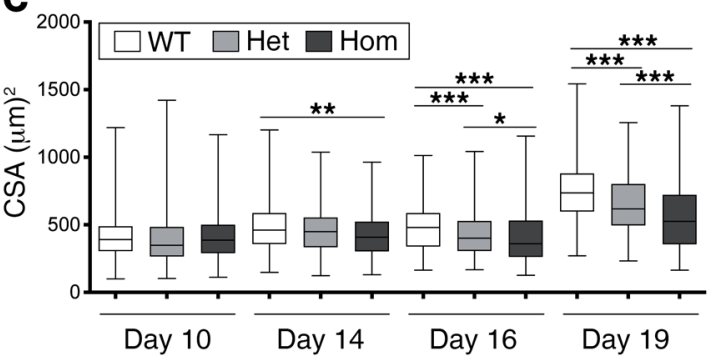

D
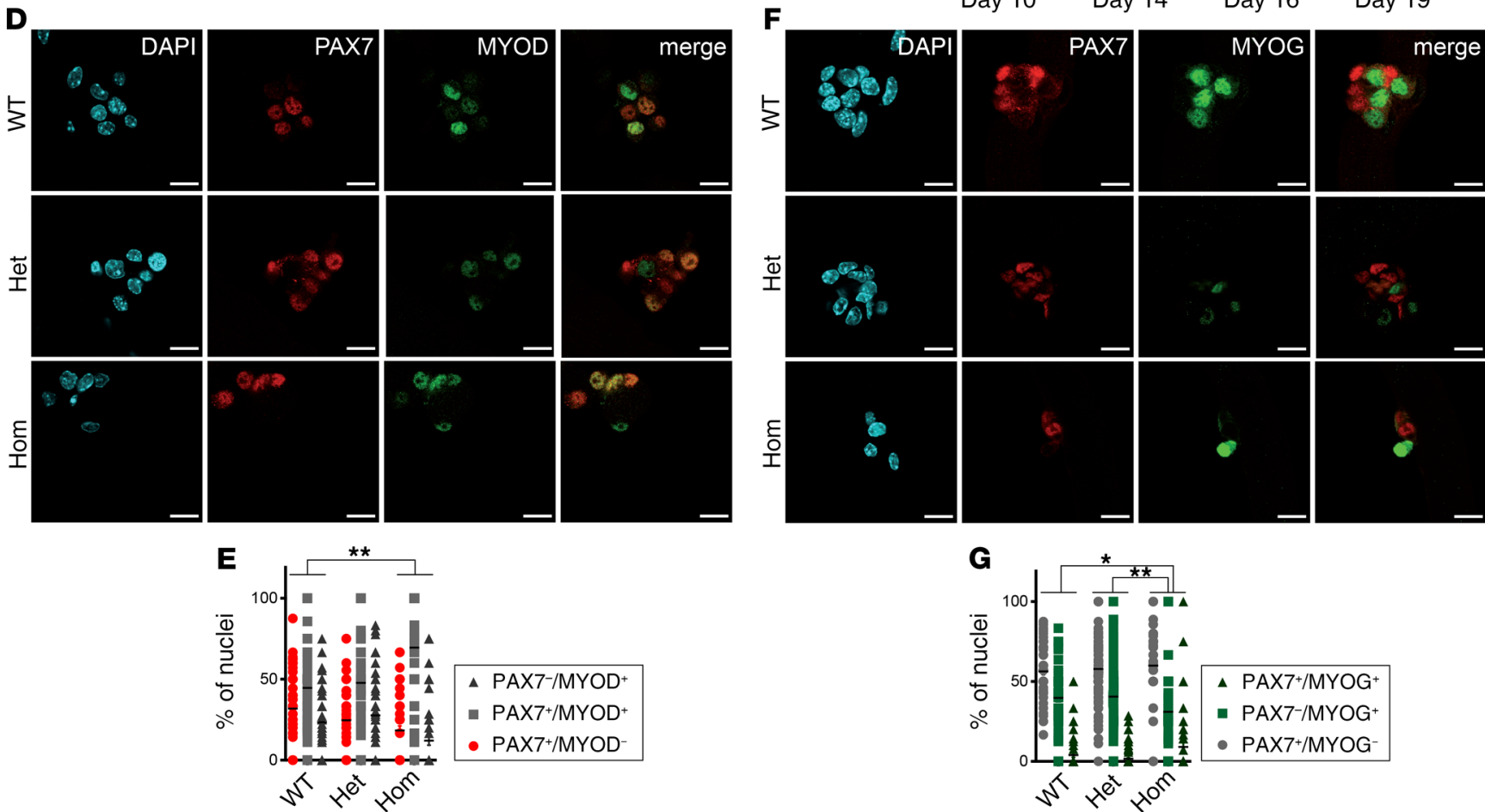

Figure 1. Lamin A regulates MuSC self-renewal. (A) Immunohistochemical staining in Lmna $\triangle 8-11$ mice of PAX7 and MYOD markers at the indicated days of postnatal growth (d10-d19). Basement membrane of muscle fibers was stained with anti-laminin. Activated, ASC (PAX7+MYOD+) and self-renewing, QSC (PAX7+MYOD') MuSCs are shown. Scale bars: $50 \mu \mathrm{m}$. (B) Quantification of MuSC pool composition in A; $n=3-6$ animals per genotype (C) Quantification of myofiber size during postnatal growth, evaluated by the cross-sectional area (CSA). $n>350$ fibers, $n=3-4$ animals per genotype. Horizontal lines within the boxes represent the medians, upper and lower bounds of the boxes represent quartiles Q3 (75th percentile) and Q1 (25th percentile), respectively, and the whiskers min to max. (D) Immunohistochemical staining of single myofibers extracted from $L$ mna $\Delta 8-11$ mice at d19 and cultured 96 hours. Activated (PAX7 $\left.7^{+} M Y O D^{+}\right)$, self-renewing (PAX7 $\left.{ }^{+} M Y O D^{-}\right)$, and differentiating (PAX7-MYOD') cells are shown. Scale bars: $20 \mu \mathrm{m}$. (E) Quantification of MuSC pool composition in $\mathbf{D} ; n>50$ muscle fibers/genotype, $n=5-8$ animals per genotype. (F) Immunohistochemical staining of single myofibers as in D. PAX7 ${ }^{+} \mathrm{MYOG}^{+}, \mathrm{PAX7}^{+} \mathrm{MYOG}^{-}$, and PAX7-MYOG ${ }^{+}$cells are shown. Scale bars: $20 \mu \mathrm{m}$. (C) Quantification of MuSC pool composition in F; $n>50$ muscle fibers/group, $n=3-5$ animals per genotype. Data in $\mathbf{B}, \mathbf{E}$, and $\mathbf{G}$ are the mean $\pm \mathrm{SEM}$. ${ }^{*} P<0.05 ;{ }^{*} P<0.01 ;{ }^{* *} P<0.001$ by 1 -way (C) or 2-way (B, E, and G) ANOVA with multiple comparisons. WT $=L m n a \Delta 8-11^{+/+}$; het $=L m n a \Delta 8-11^{+/-}$; hom $=L m n a \Delta 8-11^{-1-}$. 
A

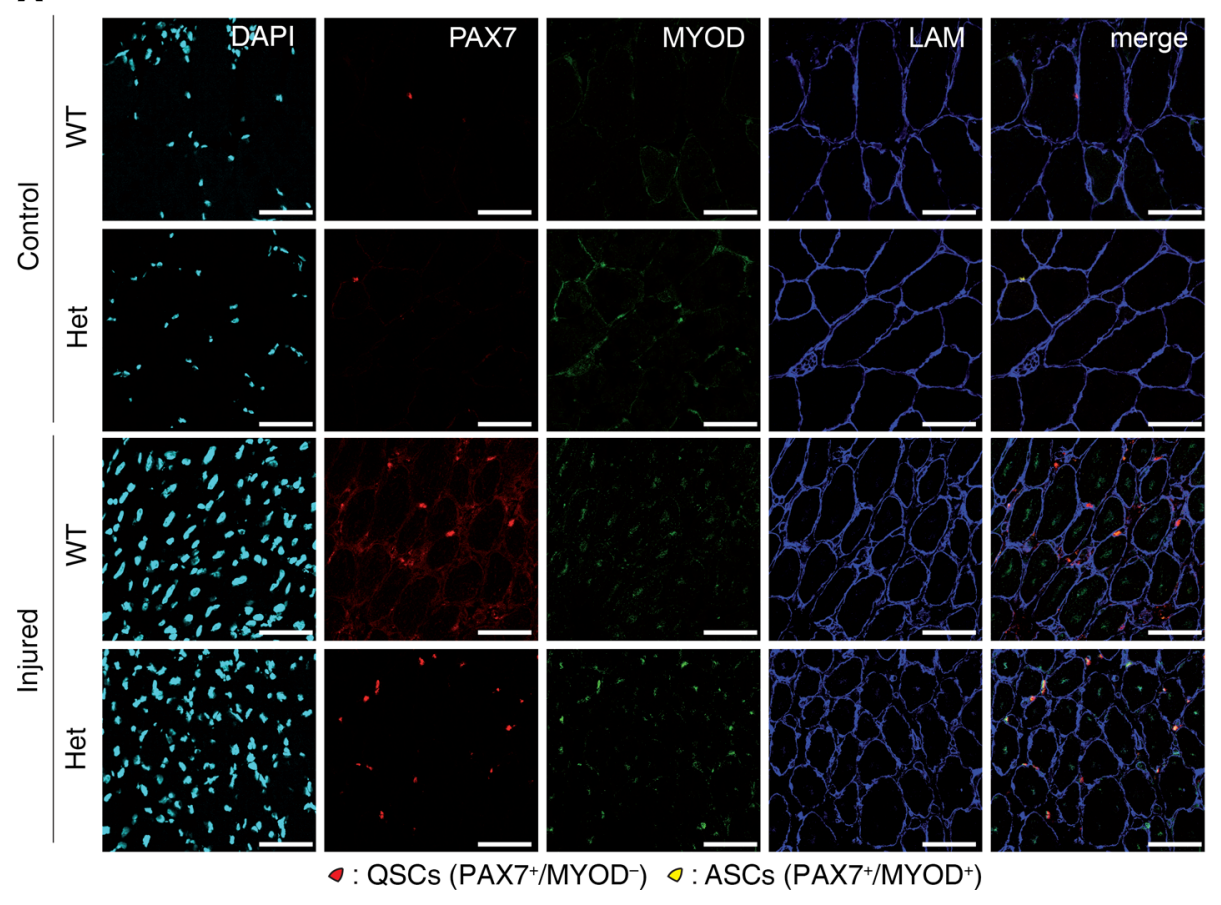

B

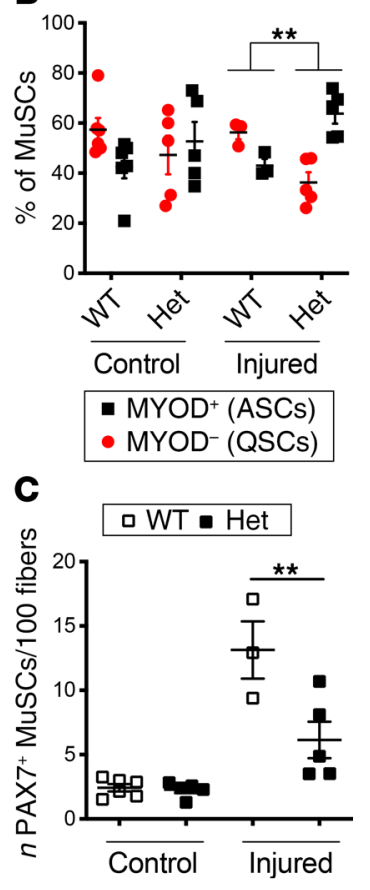

Figure 2. Lamin A levels influence muscle regeneration. (A) Immunohistochemical staining of PAX7 and MYOD markers in adult, injured $L m n a \triangle 8-11$ mice. Basement membrane of muscle fibers was stained with anti-laminin. Activated, ASC (PAX7+MYOD+) and self-renewing, QSC (PAX7+MYOD-) MuSCs are shown. Scale bars: $100 \mu \mathrm{m}$. (B) Quantification of MuSC pool composition in A; $n=3-6$ animals per group. (C) Number of Pax $7^{+}$MuSCs on 100 fibers for the experiment shown in $\mathbf{A}$. Data in $\mathbf{B}$ and $\mathbf{C}$ are the mean \pm SEM. Statistics by 1-way (C) or 2-way (B) ANOVA with multiple comparisons. Statistical comparisons between controls and injured (B and $\mathbf{C}$ ) are not shown. ${ }^{* *} P<0.01 ; \mathrm{WT}=L \mathrm{mna} \Delta 8-11^{+/+}$; het $=L m n a \Delta 8-11^{+/-}$.

supplemental material) and in whole muscle (Supplemental Figure 5, C and D; see complete unedited blots in the supplemental material). In contrast, a deep analysis of H3K27me3 ChIP-seq read coverage in the intergenic genomic regions between the known H3K27me3 enrichment peaks interestingly showed a higher average coverage in the Lmna $\Delta 8^{-11^{--}}$MuSCs compared with WT counterparts (Figure 3D). These results are compatible with a diffusion of PcG proteins along the chromatin fibers rather than a complete PcG displacement. To identify the PcG targets mostly affected by lamin A deficiency, genes were grouped according to their transcription level in WT MuSCs. We thus defined 4 equally sized groups of genes based on expression level quartiles (Figure $3 \mathrm{E})$. For each expression category, we reanalyzed the H3K27me3 distribution along the body of genes and at the TSS and the percentage of upregulated genes in the Lmna $\Delta 8-11^{-/-}$MuSCs (Figure 3 , E and F, and Supplemental Figure 5E). In quartile I we found only

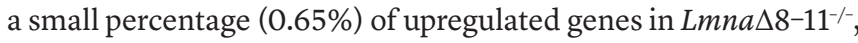
suggesting that the $\mathrm{H} 3 \mathrm{~K} 27 \mathrm{me} 3$ decrease/redistribution is not sufficient to activate transcription in highly repressed genes (Figure 3F, Supplemental Figure 5E, and Supplemental Figure 6A). In contrast, quartiles II, III, and IV are more affected by the diminished H3K27me3 levels in Lmna $\Delta 8$-11-/- (Figure 3F, Supplemental Figure $5 \mathrm{E}$, and Supplemental Figure 6B), showing a percentage of upregulated genes between $5 \%$ and $9 \%$. Specifically, we noticed that in WT MuSCs, H3K27me3 ChIP-seq signal enrichment around the TSS and within the body of genes is progressively lower in quartiles of higher expression, as expected (Figure 3F, quartiles III and IV, and Supplemental Figure 5E). However, for Lmna $\Delta 8^{-11^{-/-} \text {mice }}$ the decrease in H3K27me3 signal inside the gene body is relatively less marked than in WT mice; in fact, the average enrichment is slightly higher. We quantified and confirmed this observation by considering the ratio of $\mathrm{H} 3 \mathrm{~K} 27 \mathrm{me} 3 \mathrm{ChIP}$-seq enrichment signal at the TSS and $2.5 \mathrm{~kb}$ downstream of the TSS, for each gene, in WT and Lmna $\Delta 8^{-11^{-/}}$mice (Supplemental Figure 7A), showing that this ratio is significantly different for higher expression quartiles (Supplemental Figure 7B).

Lamin A-dependent $P c G$ redistribution determines derepression of non-muscle-related bivalent genes. The altered PcG binding observed in Lmna $\Delta 8-11^{-/}$MuSCs prompted us to examine in greater detail the bivalent genes, a subgroup of PcG targets whose expression is more susceptible to variations in PcG occupancy (27). Bivalent genes are characterized by the concurrent presence of both $\mathrm{H} 3 \mathrm{~K} 27 \mathrm{me} 3$ and H3K4me3 marks around TSSs and have an intermediate gene expression state (28). We first performed H3K27me3 and H3K4me3 ChIP-seq in WT MuSCs (Supplemental Figure 8, A and B) and we defined bivalent genes using the parameters described in Bernstein et al. (17) for the H3K4me3 window at the TSS (Supplemental Figure 8C). Then, we tested the association between bivalent and upregulated genes in the Lmna $\Delta 8-11^{-/}$MuSCs by means of Fisher's exact test. We observed a significant overrepresentation of bivalent genes among upregulated ones in the Lmna $\Delta 8-11^{-/-}$MuSCs (Figure 4A). To gain more insight into the biological relevance of deregulated genes in the mutant mice, we performed semantic similarity analysis of all Gene Ontology (GO) terms associated with upregulated genes (Figure 4B) together with GSEA (Supplemental Figure 8, D and E). These analyses showed a negative correlation with muscle 


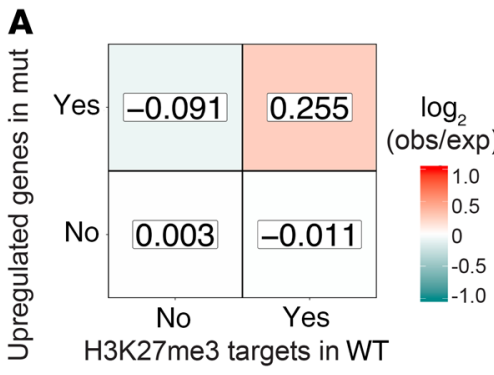

D

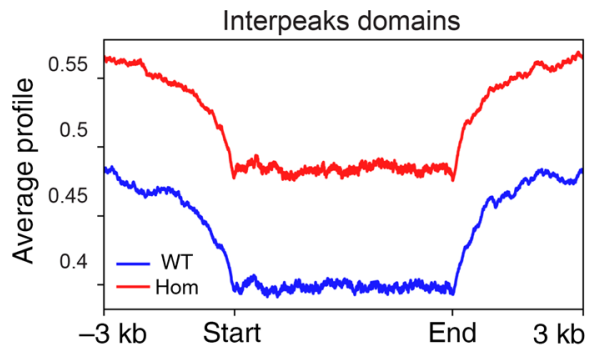

C

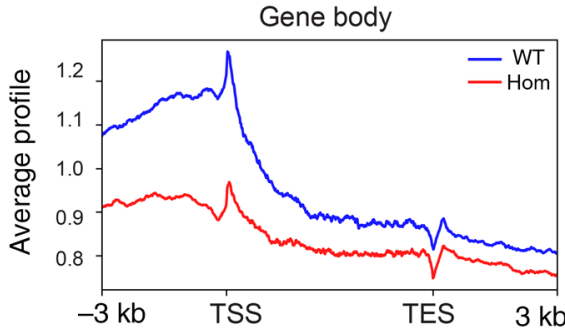

E

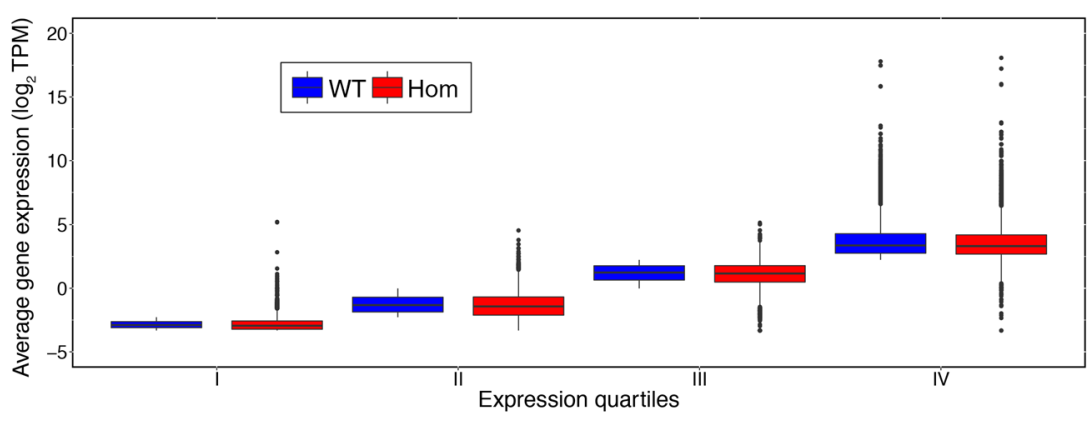

I-First quartile II-Second quartile III-Third quartile IV- Fourth quartile

$\mathbf{F}$

III

IV

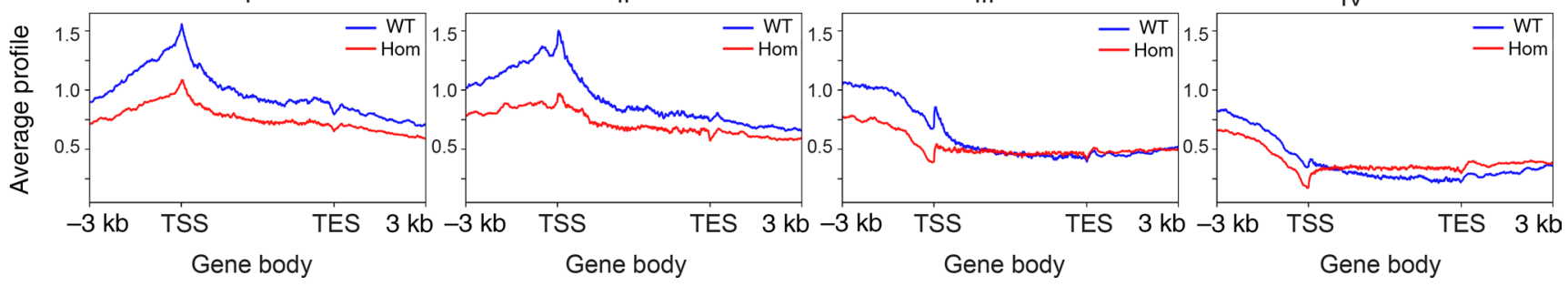

Figure 3. Lmna $\Delta$ 8-11/-- dystrophic MuSCs display PcG displacement. (A) Heatmap reporting log ${ }_{2}$ ratios of observed over expected (colored bar) number of genes in the intersections between H3K27me3 targets identified in Lmna $\Delta 8-11^{+/+}$mice and the upregulated genes in $L \mathrm{mna} \Delta 8-11^{-/-}$mice. Fisher's exact test $P=2.38 \times 10^{-5}$. (B-D) Average profile of H3K27me3 ChIP-seq signal calculated as the IP/input ratio over annotated mouse genes. (B) Average profile of H3K27me3 signal around the TSS. (C) Average profile of H3K27me3 signal along the gene body. TES, annotated transcript end. (D) Average profile of H3K27me3 signal in regions outside H3K27me3 peaks and outside annotated genes. (E) Normalized expression distribution of genes stratified using WT expression level in the 3 biological replicates (see Supplemental Methods). Distribution of average $\log _{2}$ transcripts per million (TPM + 0.1 ) values is plotted for WT and hom. Data in the boxes extend from the 25th to the 75th percentiles with the median indicated. The upper whisker extends from the hinge to the highest value that is within $1.5 \times$ IQR of the hinge, where IQR is the interquartile range, or distance between the first and third quartiles. The lower whisker extends from the hinge to the lowest value within $1.5 \times$ IQR of the hinge. Data beyond the end of the whiskers are outliers and plotted as points. (F) Average profile of H3K27me3 signal (IP/input) along the gene body using gene categories as in $\mathbf{E}$. WT $=L \mathrm{mna} \Delta 8^{-11^{+/+}}$; hom $=L \mathrm{mna} \Delta 8^{-11^{-/-}}$.

specification (Supplemental Figure 8D) together with an acquisition of markers related to lipid metabolic processes (Figure $4 \mathrm{~B}$ and Supplemental Figure 8E). Notably, Fisher's exact test analysis highlighted a significant overlap between genes with bivalent promoters and the Lmna $\Delta 8^{-11^{-/}}$MuSC upregulated genes involved in adipogenesis (Figure $4 \mathrm{C}$ ), suggesting that lamin $\mathrm{A}$ is involved in preserving MuSC identity by ensuring the correct PcG-mediated transcriptional repression of non-muscle genes.

PPAR $\gamma$ is aberrantly expressed in Lmna $48-11^{-/-}$dystrophic MuSCs. Given this strong association between bivalent gene reactivation and adipogenesis markers (Figure 4C), we analyzed different lipid-related GO categories, finding among the top GO terms peroxisome proliferator-activated receptor $\gamma$ (Pparg) (Supplemental Table 1). This master transcription factor for adipose cell differentiation $(29,30)$ was found to be significantly upregulated in Lmna $\Delta 8^{-11^{-/}}$MuSCs (FDR $<0.05$ ). Moreover, the Pparg gene is a polycomb target and has a bivalent sig- nature in WT MuSCs (Supplemental Table 1 and Supplemental Figure 9A). These observations prompted us to analyze Pparg transcriptional deregulation. We stained muscles for PAX7 and PPAR $\gamma$ to directly test if MuSCs displayed aberrant expression of PPAR $\gamma$ in the absence of lamin A. Strikingly, we found approximately $10 \%$ of $L m n a \Delta 8^{-11^{-/}}$ MuSCs that simultaneously express both muscular and adipogenesis markers, being $\mathrm{PAX} 7^{+} \mathrm{PPAR} \gamma^{+}$(Figure 5, A and B). Accordingly, the genomic region of the Pparg gene showed a decrease in H3K27me3 enrichment around the TSS in the Lmna $\Delta 8^{-11^{--}}$MuSCs, accompanied by transcriptional upregulation (Supplemental Figure 9B). To evaluate if the aberrant expression of adipogenic genes in MuSCs of mutant mice culminates with fatty infiltration we performed immunofluorescence staining for perilipin 1, a protein present on the surface of lipid droplets (ref. 31 and Figure 5, C and D), on cryosections of muscles derived from d19 Lmna $\Delta 8-11$ mice. We found large areas of adipose accumulation between myofibers of lamin 


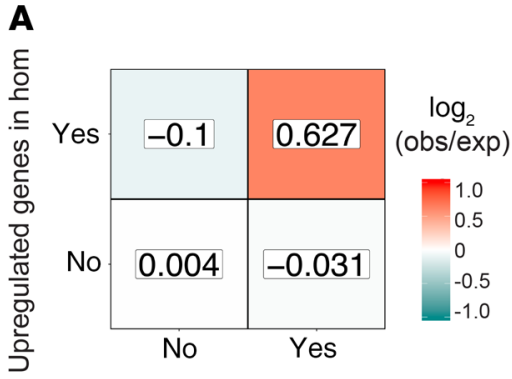

Genes with bivalent promoter

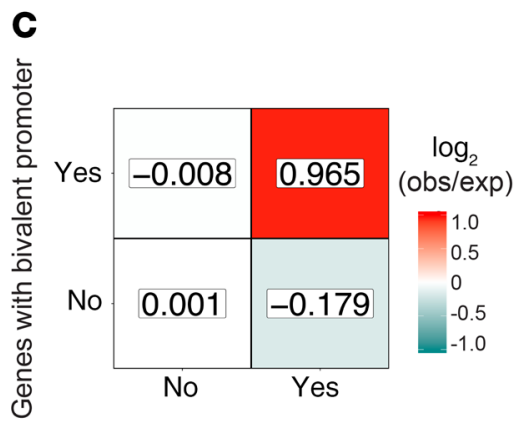

Adipogenesis upregulated genes
B

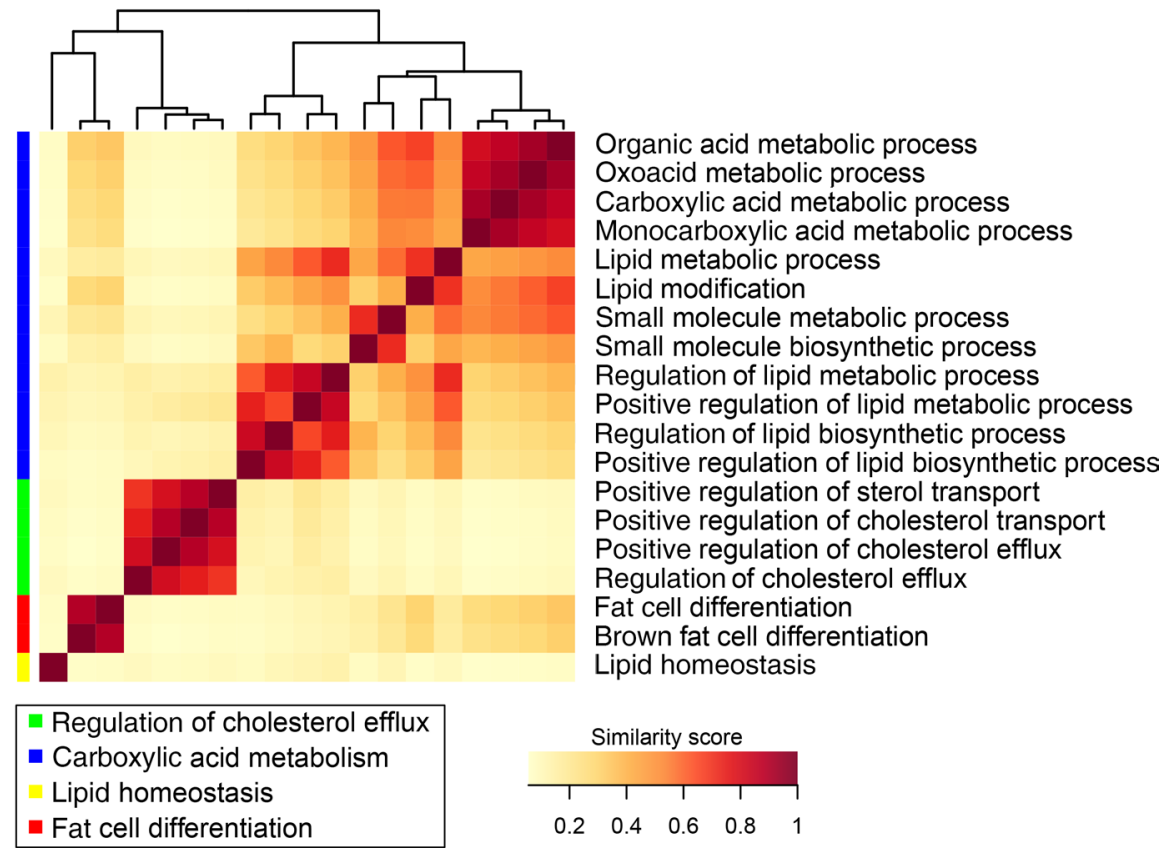

Figure 4. Lamin A-PcG-mediated transcriptional repression preserves MuSC identity. (A) Heatmap reporting log ${ }_{2}$ ratios of observed over expected number of genes (colored bar) in the intersections between bivalent promoters identified in WT satellite cells and the upregulated genes in $L m n a \Delta 8-11^{-/-}$mice. Fisher's exact test $P=4.57 \times 10^{-7}$. (B) Semantic similarity analysis of GO terms enriched in upregulated genes in hom versus WT comparison (FDR $<0.05$ ) with macrocategories identified using the REVIGO web tool (http://revigo.irb.hr/). (C) Heatmap reporting $\log _{2}$ ratios of observed over expected number of genes (colored bar) in the intersections between upregulated genes in $L m n a \Delta 8-11^{-/-}$mice in Pparg-related GO terms and the bivalent genes identified as above. Fisher's exact test $P=6.73 \times 10^{-6}$. WT $=L m n a \Delta 8-11^{+/+} ;$hom $=L m n a \Delta 8-11^{-1-}$.

$\mathrm{A} / \mathrm{C}^{+/}$and lamin $\mathrm{A} / \mathrm{C}^{-/-}$muscles, which were instead undetectable in the WT mice. Considering the key role of PcG proteins in mediating the formation of chromatin loop structures $(32,33)$, we reasoned that the loss of H3K27me3 and transcriptional upregulation of the Pparg locus could be related to the alteration of chromatin 3D structure. The genomic 3D architecture is organized in structurally separated topologically associated domains (TADs), chromosomal structures that favor intradomain looping interactions (34). TADs can be identified by genome-wide chromosome conformation capture (Hi-C) and are largely conserved across different cell types. We verified that the Pparg locus is included in a TAD encompassing a region extending also upstream of the Pparg locus itself, using high-resolution Hi-C data on mouse embryonic stem cells (35) and the 3D Genome Browser (ref. 36 and Supplemental Figure 10). Then, we performed 3D multicolor DNA FISH analysis on prefixed MuSCs using $1 \mathrm{BAC}$ probe overlapping the Pparg promoter and a second probe annealing at the other TAD border. We observed an overlap of the signals from the 2 regions in the WT Lmna $\Delta 8-11^{+/+}$MuSCs that indicates the presence of a DNA looping in cis (Figure 5, E and F). By contrast, in Lmna $\Delta 8^{-11^{-/-}}$ MuSCs the distance between the signals was higher, definitely suggesting the lack of DNA/DNA interactions. Indeed, from the analysis of H3K27me3 ChIP-seq tracks we noticed in the Lmna $\Delta 8-11^{--}$MuSCs a reduction in H3K27me3 peaks upstream of the Pparg locus (Supplemental Figure 10). FISH analysis also highlighted that in WT the entire genomic region is close to the nuclear periphery (Figure 5, E and G), whereas in Lmna $\Delta 8-11^{-/-}$it is relocated in the nuclear interior, suggesting that lamin $\mathrm{A}$ absence interferes with chromatin anchoring to the nuclear lamina and PcG-dependent DNA conformation.
Lmna 8-11/- MuSCs undergo premature senescence. Taken together, these results clearly point toward a role of lamin A in mediating PcG-transcriptional repression in MuSCs to safeguard their identity and regenerative capacity. This lack of cell identity and the impairment of self-renewal displayed by Lmna $\Delta 8-11^{-/-}$MuSCs are all features reminiscent of the phenotype described for Ezh2-null MuSCs (24). Moreover, the impairment in self-renewal and the progressive decline of the MuSC pool are also typical traits of aged MuSCs (37) in which both lamin A/C and PcG proteins play a key role $(38,39)$. A major cellular mechanism that ensures self-renewal and hence the maintenance of the MuSC pool is asymmetric division (40). At the molecular level, in aged mice, the accumulation of the activated form of p38 (phospho-p38 [p-p38]) and its symmetric distribution in MuSC doublets heavily compromise the selfrenewal capacity, leading to MuSC functional decline (41, 42). To test whether premature exhaustion of quiescent Lmna $\Delta 8^{-11^{-/}}$ MuSCs could be ascribed to defective asymmetric division, we stained myofiber-associated MuSCs for p-p38 after 48 hours of culture, a timing at which d19 myofibers formed MuSC-derived doublets (Figure 6A). In contrast to WT, Lmna $\Delta 8-11^{-/}$MuSC doublets showed a preferential symmetric distribution of p-p38, quantified by relative fluorescence intensity (Figure 6, A and B), often accompanied by a planar orientation with respect to myofibers (see Supplemental Methods and Figure 6C). This highlights problems in asymmetric division, which should be instead characterized by apico-basal orientation (43). In line with this result, in Lmna $\Delta 8-11^{-/-}$muscle sections we found higher amounts of p-p38 (Supplemental Figure 11, A and B) MuSCs and signs 
A

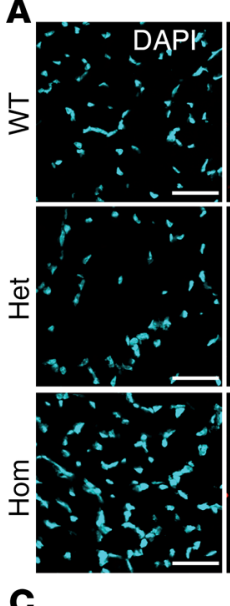

C

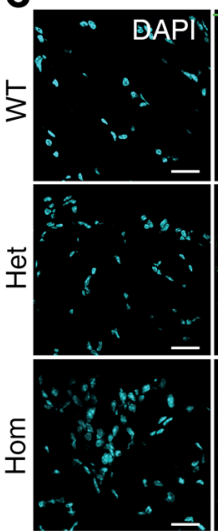

E

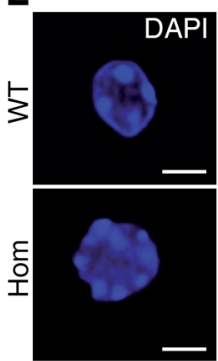

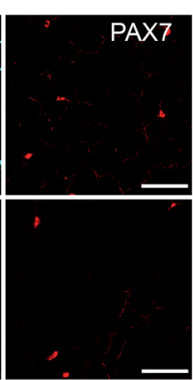
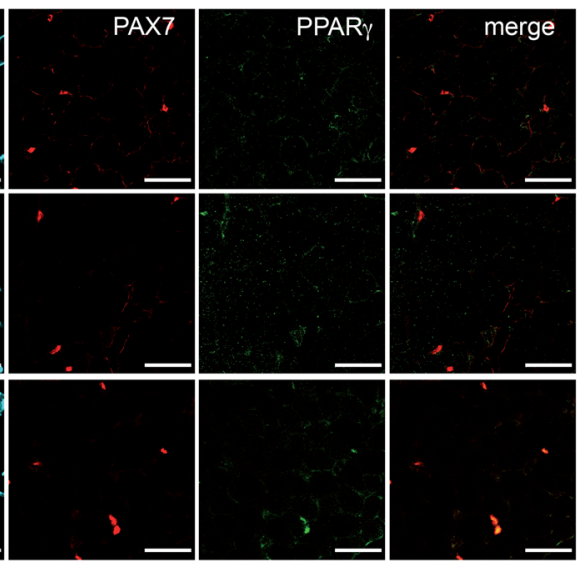

D
B

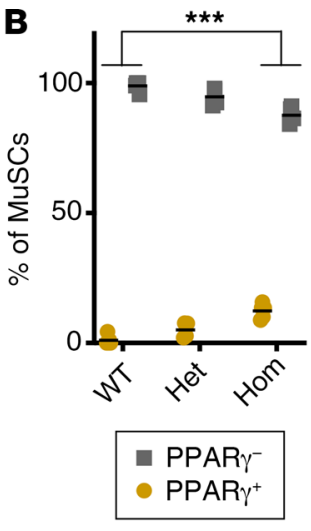

Figure 5. Deregulation of Pparg locus in Lmna $\Delta$ 8-11-/- MuSCs. (A) Immunohistochemical staining of PAX7 and PPAR $\gamma$ markers in Lmna $\Delta 8-11$ mice at 19 days of postnatal growth (d19). Scale bars: $50 \mu \mathrm{m}$. (B) Quantification of PPAR $\gamma^{+}$MuSCs in A; $n=4-5$ animals per genotype. Data are the mean \pm SEM. (C) Immunohistochemical staining of perilipin (PLIN) in Lmna $\Delta 8-11$ muscles at d19. Nuclei of muscle fibers were stained with DAPI. Scale bars: $25 \mu \mathrm{m}$. (D) Quantification of perilipin staining in $\mathbf{C} ; n=5$ animals per genotype. (E) Representative image of FISH analysis of fixed and sorted MuSCs from Lmna $\Delta 8-11$ mice at d19 with probes indicated in Supplemental Figure 10. Scale bars: $2 \mu \mathrm{m}$. (F) Quantification of FISH probe (represented in Supplemental Figure 10) distances ( $x$ axis) versus cumulative frequency distributions ( $y$ axis). Only probes with distances of $0.5 \mu \mathrm{m}$ or less are reported. $n=1-2$ animals per genotype. (C) Quantification of FISH probe position with respect to the nuclear envelope. In the box-andwhisker plots in $\mathbf{D}$ and $\mathbf{G}$, horizontal lines within the boxes represent the medians, upper and lower bounds of the boxes represent quartiles Q3 (75th percentile) and Q1 (25th percentile), respectively, and whiskers min to max. ${ }^{*} P<0.05$; ${ }^{* *} P<0.001$ by 2 -way ANOVA with multiple comparisons (B and $\mathbf{D})$ or Kolmogorov-Smirnov test ( $\mathbf{F}$ and $\mathbf{G}$ ). WT $=L m n a \Delta 8-11^{+/+}$; het $=L m n a \Delta 8-11^{+/-}$; hom $=$ Lmna $\Delta 8-11^{-1-}$.

of genomic instability, as measured by increased $\gamma \mathrm{H} 2 \mathrm{AX}$ DNA repair signal foci (Supplemental Figure 11, C and D), not accompanied by apoptosis or necrosis as evidenced by annexin staining (Supplemental Figure 12A). To test if defective asymmetric division is associated with premature senescence we then analyzed RNA-seq to determine if Lmna $\Delta 8^{-11^{-/-}}$MuSCs share the same transcriptional signature of MuSCs isolated from aged mice. We performed a GSEA using 2 different RNA data sets from MuSCs of 24-month-old mice $(25,44)$ and $L m n a \Delta 8-11^{-/-}$upregulated genes. In line with our hypothesis, we found that d19 Lmna $\Delta 8^{-11^{-1}}$ MuSCs present an upregulated transcriptome similar to 20- to 24-month-old MuSCs (Figure 6D and Supplemental Figure 12B), but different from geriatric 28- to 32-month-old MuSCs (Supplemental Figure 12C). At the molecular level, the senescence program is supported by upregulation of some PcG-regulated cyclindependent kinase inhibitors (CDKIs) (45) such as p21, which is involved in cellular senescence and in cell cycle arrest. p21 maintains the viability of DNA damage-induced senescent cells (46) and aberrant expression of p21 has been observed in EDMDderived human myoblasts (47). ChIP-seq and RNA-seq analyses of the Cdkn1a/p21 locus showed a displacement of Ezh2 from the promoter accompanied by an upregulation of p21 transcripts in Lmna $\Delta 8-11^{-/-}$MuSCs (Figure 6E).

Genetic ablation of the Cdkn2a locus partially rescues selfrenewal defects in Lmnas8-11/- dystrophic mice. To further corroborate our findings, we also analyzed the Cdkn2a locus, a PcG target primarily involved in muscular senescence (ref. 44 and Supplemental Figure 13A). Two transcripts, p16 ${ }^{\mathrm{INK} 4 \mathrm{a}}$ and $\mathrm{p} 19^{\mathrm{ARF}}$, originate from the Cdkn2a locus (48). Interestingly, it was recently reported that $\mathrm{p} 16^{\mathrm{INK} 4 \mathrm{a}}$ expression is a second event, subsequent to p21 upregulation, in cellular senescence progression (49). In line with these observations, $\mathrm{p} 16^{\mathrm{INK} 4 \mathrm{a}}$ expression is specifically induced in geriatric 28- to 32-month-old MuSCs (but not in 24-month-old MuSCs) (44). Moreover, depletion of p16 ${ }^{\mathrm{INK} 4 \mathrm{a}}$ is sufficient to reduce senescence-associated gene expression in geriatric MuSCs. RNA-seq and quantitative reverse transcription 


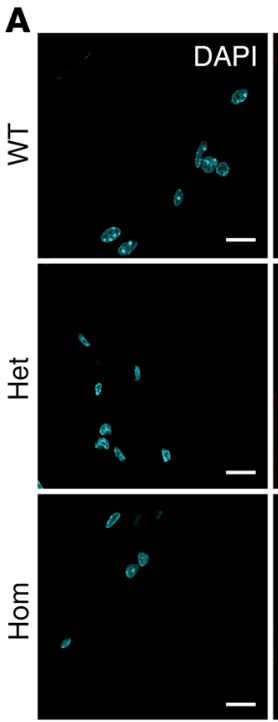

D

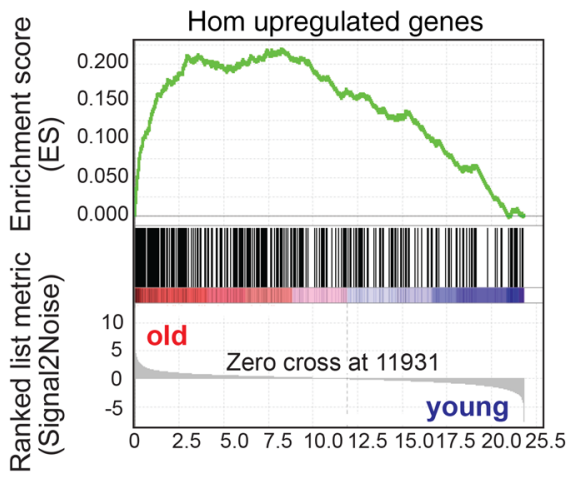

Rank in Ordered Dataset $\left(\times 10^{3}\right)$ -Enrichment profile - Hits - Ranking metric scores

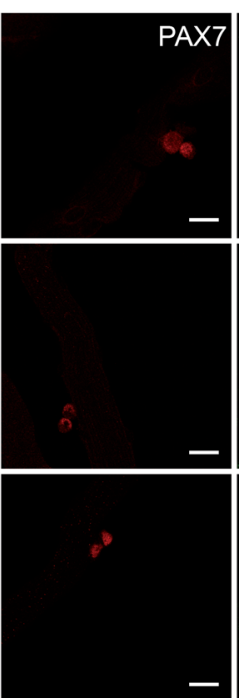

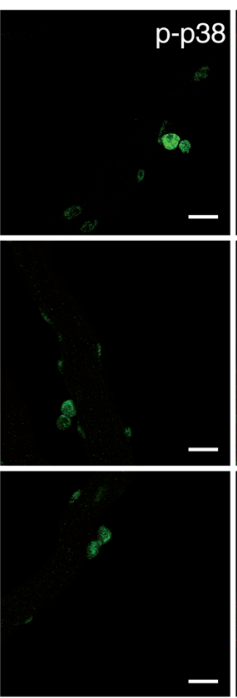
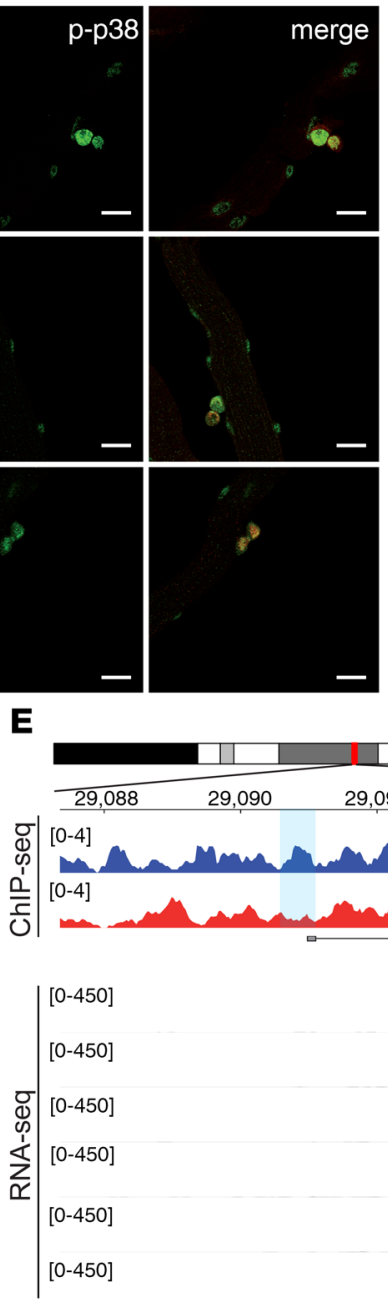
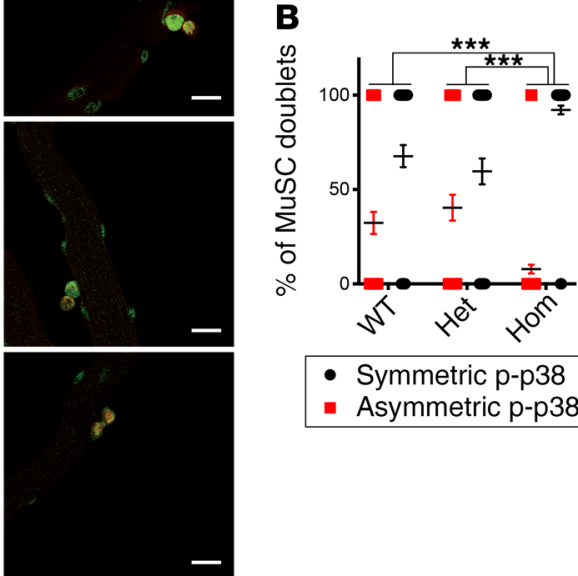

Symmetric p-p38

- Asymmetric p-p38

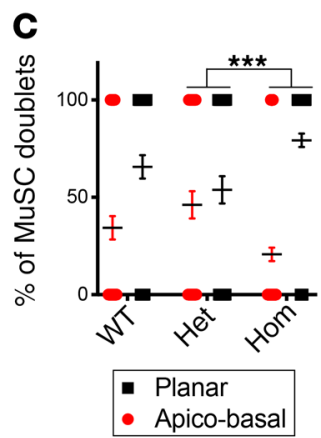

Chromosome 17

Figure 6. Lmna $\Delta 8-11^{-/-}$MuSCs acquire senescence transcriptional traits. (A) Representative image of myofiber-derived MuSCs from Lmna $\Delta 8-11$ mice at d19 immunostained for p-p38 and PAX7 after 48 hours of culture. Scale bars: $25 \mu \mathrm{m}$. (B) Quantification of asymmetric and symmetric divisions assessed by p-p38 distribution as shown in A. (C) Quantification of asymmetric apico-basal division versus symmetric planar divisions. $n=46 \pm 6$ doublets of MuSCs per genotype, $n=7-9$ mice per group. Data in $\mathbf{B}$ and $\mathbf{C}$ are the mean \pm SEM. (D) GSEA of expression data from old and young mouse quiescent satellite cells (25). Upregulated (log[fold change] >1) genes in hom versus WT comparison added to Biocarta mouse pathways from the gskb R package were used as gene sets (NES $\left.=4.70, F D R<1 \times 10^{-4}\right)$. (E) ChIP-seq of H3K27me3 mark and RNA-seq signal tracks on the Cdkn1a/p21 locus. Promoter regions are highlighted by light blue rectangles. Statistics by 2-way ANOVA with multiple comparisons. ${ }^{* *} P<0.01 ;{ }^{* *} P<0.001$. WT $=L m n a \Delta 8-11^{1+}$; het $=L m n a \Delta 8-11^{+/-}$; hom $=L m n a \Delta 8-11^{-/-}$.

PCR (qRT-PCR) on d19 Lmna $\Delta 8^{-11^{-/-}}$MuSCs did not reveal any transcription of $\mathrm{p} 16^{\mathrm{INK} 4 \mathrm{a}}$ in WT or Lmna $\Delta 8^{-11^{-/-}}$(Figure 7A). However, qRT-PCR analysis performed in older mice (d26) revealed higher levels of p16 ${ }^{\mathrm{INK} 4 \mathrm{a}}$ transcripts in Lmna $\Delta 8^{-11^{-/-}}$MuSCs and whole muscles compared with the WT counterpart (Figure 7A), suggesting a transition during dystrophy progression toward a geriatric condition. We thus decided to test whether genetic ablation of the Cdkn2a locus could reverse Lmna $\Delta 8^{-11^{-/-}}$MuSC

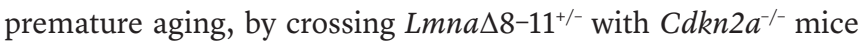
(50). Analysis of the Lmna $\Delta 8-11^{+/+}$background showed no differences in the percentages of QSCs and ASCs, nor in CSA, suggesting that $C d k n 2 a$ is dispensable for postnatal muscle development (Figure 7, B-D; $L M N A^{+/+}$). On the other hand, $C d k n 2 a^{-/-} L m n a \Delta 8-11^{-/-}$mice partially rescued the quiescent MuSC pool and CSA defects observed in the absence of lamin A (Figure 7, B-D; $L M N A^{-/}$; Supplemental Figure 13, B and C), emphasizing that lamin A-dependent muscular dystrophy might be due to progressive MuSC functional decline caused by acquisition of premature aging features.

\section{Discussion}

Lamin A-dependent muscular dystrophy pathogenesis has been classically ascribed to nuclear fragility that renders myonuclei more prone to mechanical stress and damage imposed by myofiber contraction (4). However, the evidence that lamin A/C is expressed also by MuSCs has led to the suggestion that satellite cell dysfunction might contribute to EDMD progression (51), yet experimental evidence in support of this hypothesis is still lacking.

Cell fate choice during muscle differentiation is governed by epigenetic factors controlling the sequential restriction of transcriptional programs (52). Any dysfunction in this finely tuned epigenetic regulation could lead to impaired or aberrant cell fate determination (53). 

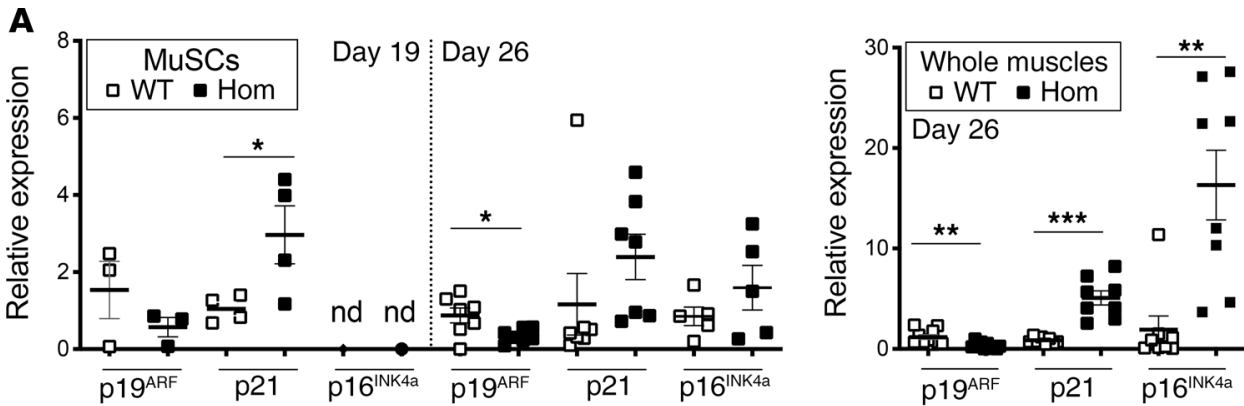

B

$L M N A^{+/+}$
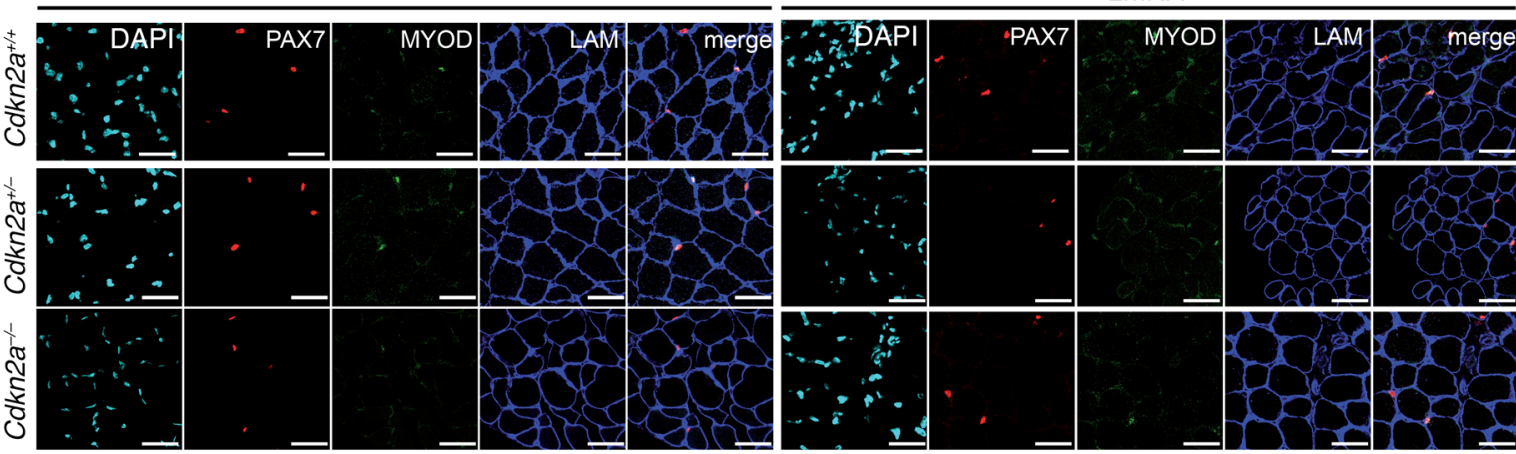

: QSCs (PAX7'MYOD-)

$\circ:$ ASCs (PAX7+MYOD-)

C
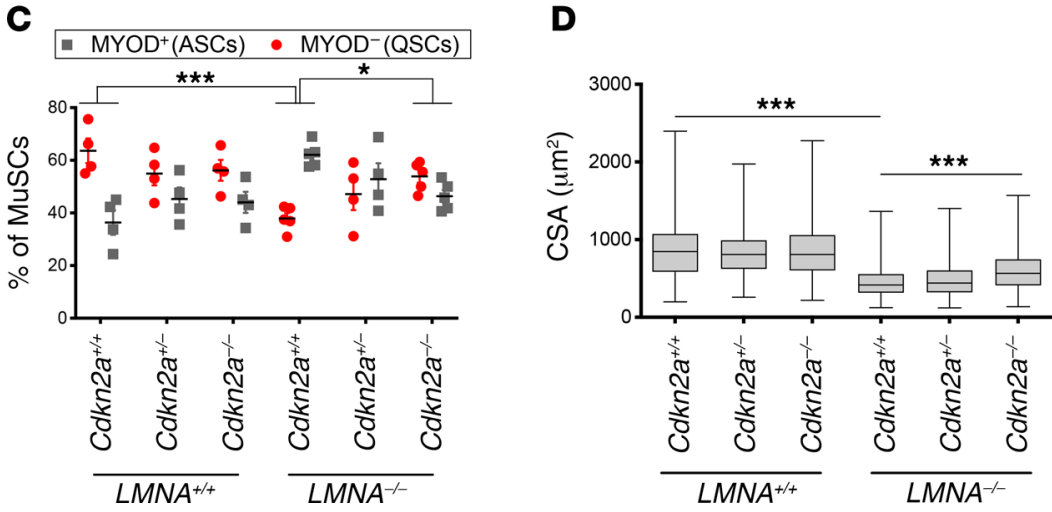

Figure 7. Cdkn2a genetic ablation restores regenerative capacity of $L m n a \Delta 8-11^{-/-}$dystrophic mice. (A) Transcriptional analysis of p16 ${ }^{I N K 4}$ and p19ARF at the Cdkn2a locus in Lmna $\Delta$ 8-11 mouse MuSCs (left graph) at d19 and d26 and whole muscles (right graph) at d26. Values were normalized to Gapdh and compared with the average of WT amplification. nd, not detected. $n=3-10$ animals per genotype. (B) Immunohistochemical staining of PAX7 and MYOD markers in Cdkn2a/Lmna $\triangle 8-11$ mice at d19. Basement membrane of muscle fibers was stained with anti-laminin. Activated, $A S C$ (PAX7 $\left.7^{+} / \mathrm{MYOD}^{+}\right)$and self-renewing, QSC (PAX7+MYOD-) MuSCs are shown. Scale bars: $50 \mu \mathrm{m}$. (C) Quantification of MuSC pool composition in B. $n=4-5$ animals per genotype. Data in $\mathbf{A}$ and $\mathbf{C}$ are the mean \pm SEM. (D) Quantification of myofiber size, evaluated by the cross-sectional area (CSA). $n=600$ muscle fibers. $n=4-5$ animals per genotype. The horizontal lines within the boxes represent the medians, upper and lower bounds of the boxes represent quartiles $\mathbf{Q} 3$ (75th percentile) and Q1 (25th percentile), respectively, and whiskers min to max. ${ }^{*} P<0.05 ;{ }^{*} P<0.01$; ${ }^{* *} P<0.001$ by unpaired $t$ test (A) or by 1-way (D) or 2-way (C) ANOVA with multiple comparisons. WT $=L m n a \Delta 8-11^{+/+}$; hom $=L m n a \Delta 8-11^{-1-}$.

Here, we show that lamin A/C is indeed crucial to preserve MuSC identity and regenerative capacity. We demonstrate that cell-autonomous lamin A-dependent polycomb dysfunction leads to MuSC functional decline, which culminates with impaired regenerative capacity and a dystrophic phenotype (Figure 1). Traditionally, the role of lamin $\mathrm{A} / \mathrm{C}$ in muscle differentiation has been considered to cause defects in muscle differentiation $(54,55)$. However, in other conditions, MuSCs from lamin A/C-null mice showed a normal ability to differentiate and to form myotubes $(20,56)$. By moving the viewpoint from differentiation to cell identity we now propose that in the absence of lamin $\mathrm{A} / \mathrm{C}$, a portion of MuSCs are derailed from their fate, affecting the quiescent MuSC pool. At the molecular level, we describe a mechanism of lamin A-dependent deregulation of PcG targets showing the spreading of repressive marks along the chromatin fiber (Figure 3), with lack of the expected higher order structures and consequent derepression of bivalent genes (Figures 4, 5, and 8). Recently, it has been proposed that PcG domains can have distinct size and boundary characteristics (57); upon differentiation, loci directly involved in fate specification lose PcG-mediated looping interactions, allowing new active promoter/enhancer interactions. In parallel, other PcG domains, such as the Hox clusters, do not change their 3D architecture. Our findings further corroborate this hypothesis, showing that the stability of PcG-interacting domains correlates with PcG occupancy and depends on lamin A (Figures 5 and 8). PcG 

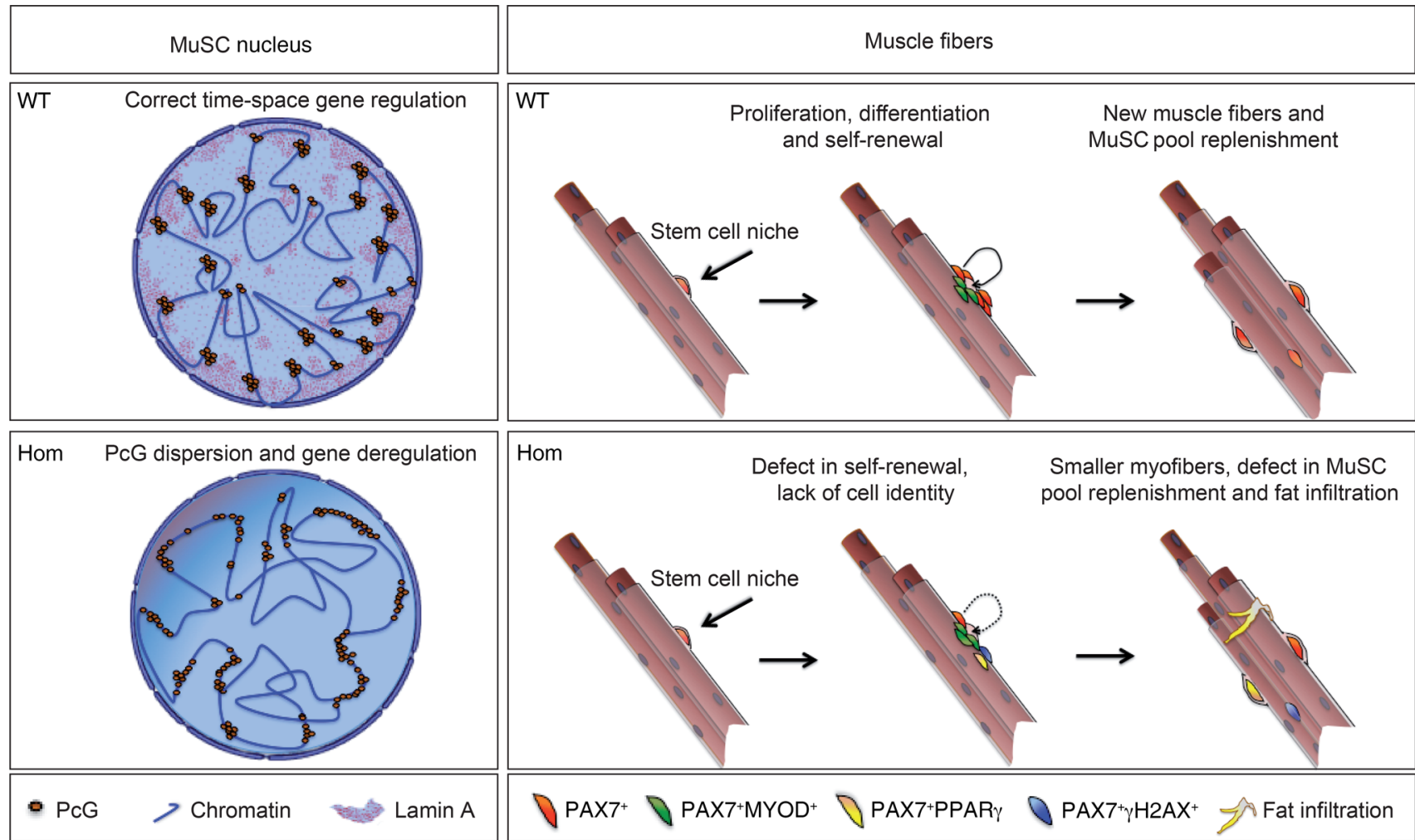

Figure 8. Lamin A/C-polycomb crosstalk in lamin-dependent muscular dystrophy. In WT MuSCs the lamin A-PcG interplay sustains the chromatin higher order structure at differentiation loci, ensuring proper spatio-temporal gene regulation during muscle differentiation. The absence of lamin A/C determines PcG displacement and relaxation of PcG-mediated higher-order chromatin structure. In Lmna $\Delta 8-11^{-/-}$MuSCs lamin A/C-PcG dysfunctional crosstalk causes a lack of cellular identity and premature senescence, finally leading to an exhaustion of the stem cell niche and infiltration of adipogenic cells. WT $=L m n a \Delta 8-11^{+/+} ;$hom $=L m n a \Delta 8-11^{-1-}$.

dysfunctions drive Lmna $\Delta 8-11^{-/}$MuSCs toward 2 not mutually exclusive fates (Figure 8): lack of cell identity, highlighted by the presence of MuSCs coexpressing muscle and adipogenic markers (Figures 4 and 5); and premature senescence, as shown by defects in asymmetric division and accumulation of p-p38 and $\gamma \mathrm{H} 2 \mathrm{AX}$ (Figure 6 and Supplemental Figure 11). These epigenetic alterations determine a progressive decline in MuSC selfrenewal that accompanies the muscular dystrophy progression (Figure 1), ultimately leading to a geriatric condition characterized by the expression of $\mathrm{p} 16^{\mathrm{INK} 4 \mathrm{a}}$ from the $C d k n 2 a$ locus (Figure 7 and ref. 58). Genetic ablation of the Cdkn2a locus can recover some muscular dystrophy defects of the Lmna $\Delta 8-11^{-/-}$mouse (Figure 7), thus supporting the hypothesis that dystrophic and aging muscles share dysfunction in epigenetic mechanisms controlling cell cycle and fate decisions of MuSCs. Our findings corroborate recent evidence of PcG dysfunction in human disease (19), showing that PcG alterations contribute to pathology progression and severity in EDMD. This will further stimulate future studies on the role of PcG proteins in the dynamics of the stem cell niche, when embedded in a pathological environment.

\section{Methods}

\section{Animals}

Heterozygous B6.129S1(Cg)-Lmnatm1Stw/BkknJ mice $\left(\right.$ Lmna $\left.\Delta 8-11^{+/-}\right)$ (21) and $C d k n 2 a^{+/-}$mice (50) were used.

\section{Satellite cell extraction, apoptosis evaluation, and multiple injuries}

Hind-limb muscles were isolated from sacrificed mice and digested for 120 minutes in $2.4 \mathrm{U} / \mathrm{mL}$ Dispase II (Roche, 04942078001), $2 \mu \mathrm{g} / \mathrm{mL}$ collagenase A (Roche, 1013586001), $0.2 \mathrm{mM} \mathrm{CaCl}_{2}$ (MilliporeSigma, C5670), $4 \mathrm{mM} \mathrm{MgCl}_{2}$ (MilliporeSigma, M8266), and $10 \mathrm{ng} / \mathrm{mL}$ DNase I (Roche, 1014159001) in 1× PBS (Euroclone, ECB $4004 \mathrm{~L}$ ) at $37^{\circ} \mathrm{C}$ in a water bath. The samples were resuspended in HBSS (Gibco, 14025-050) supplemented with 0.1\% BSA (MilliporeSigma, A7030). Cell suspensions were serially filtered with 70- $\mu \mathrm{m}$ (Falcon, 352350) and 40- $\mu \mathrm{m}$ filters (Falcon, 352340), stained with antibodies indicated in Supplemental Table 2 (PB-CD45 1:50, PB-CD31 1:50, PB-Ter119 1:50, FITC-Sca1 1:50, APC- $\alpha$ 7integrin 1:200), and sorted with a BD FACSAria III for PB-CD45 ${ }^{-} \mathrm{PB}-\mathrm{CD} 31^{-}$ PB-Ter119-FITC-Sca1-APC- $\alpha$ 7integrin ${ }^{+}$.

For multiple-injury experiments, $20 \mu \mathrm{L}$ of $10 \mu \mathrm{M}$ cardiotoxin (CTX) (Latoxan, L8102) was injected in the tibialis anterior (TA) muscle each week for 3 weeks. TA muscle was harvested 1 week after the last CTX injection.

For apoptosis assays we stained MuSCs with annexin. Satellite cells $\left(1.5 \times 10^{4}\right.$ to $\left.20 \times 10^{4}\right)$ were washed in $1 \mathrm{~mL}$ cold PBS, centrifuged 7 minutes at $400 \mathrm{~g}$, and incubated 20 minutes at room temperature in the dark with $100 \mu \mathrm{L}$ Annexin V FITC buffer (FITC Annexin V Apoptosis Detection Kit I; BD Biosciences, 556547). Then, samples were washed with $500 \mu \mathrm{L}$ of cold PBS, centrifuged 7 minutes at 400 $g$, and incubated 15 minutes at room temperature in the dark with $300 \mu \mathrm{L}$ of cold PBS containing $5 \mu \mathrm{L}$ of propidium iodide. Samples 
were analyzed on a BD FACSCanto (voltage $\mathrm{FSC}=357$, $\mathrm{SSC}=462$, medium flow to acquire, 300 to 400 events/second).

\section{Immunofluorescence}

Muscle sections. TA muscles were embedded in Killik (Bio-Optica, 05-9801), immediately frozen in precooled isopentane (MilliporeSigma, 277258), and sectioned (Leica CM1850 cryostat) at $8 \mu \mathrm{m}$. Sections were fixed 20 minutes in $4 \%$ paraformaldehyde (PFA) (MilliporeSigma, P6148) and washed 3 times, 5 minutes each in PBS. To permeabilize tissues, precooled methanol (MilliporeSigma, 322415) at $-20^{\circ} \mathrm{C}$ was added for 6 minutes. Antigen retrieval was performed 2 times, 5 minutes each in hot citric acid (MilliporeSigma, C0759) $\left(80^{\circ} \mathrm{C}, \mathrm{pH} 6.0\right)$ and washed 2 times, 5 minutes each in PBS. Sections were blocked 1 hour in 4\% BSA (MilliporeSigma, A7030) followed by incubation for 45 minutes with Fab mouse fragment 1:100 (Jackson ImmunoResearch, 115-007-003) in PBS. Primary antibodies were diluted 1:100 in blocking solution, except for anti-PPAR $\gamma$ which was diluted 1:75, and incubated overnight at $4^{\circ} \mathrm{C}$. The day after, sections were washed 3 times, 10 minutes each in PBS/0.1\% BSA and incubated with secondary antibodies diluted 1:200 in blocking solution for 1 hour at room temperature in the dark. Then, sections were washed 3 times, 10 minutes each in PBS/0.1\% BSA and incubated 2 hours at room temperature with anti-laminin and - $\operatorname{Pax} 7$ (1:20). After washing 3 times, 10 minutes each in PBS/0.1\% BSA, sections were incubated 45 minutes with anti-Cy5 (1:300) and biotin (1:500) for Pax7 signal amplification. After washing 3 times, 10 minutes each in PBS, sections were incubated 45 minutes with Cy3-streptavidin (1:1250). The sections were finally washed 3 times, 10 minutes each in PBS, stained 5 minutes with DAPI (MilliporeSigma, D9542), briefly washed twice in PBS, and mounted on slides with a drop of Prolong Antifade (Thermo Fisher Scientific, P36930).

Single myofibers. TA, soleus, gastrocnemius, and extensor digitorum longus were isolated from mice and digested 45 to 50 minutes in $0.2 \%$ collagenase type I (MilliporeSigma, C0130)/DMEM (Gibco, $10569010)$ at $37^{\circ} \mathrm{C}$. Two rounds of myofiber washes were performed in precoated dishes with 20\% FBS (Gibco, 10500064)/DMEM. Myofibers were grown in DMEM supplemented with 20\% FBS, $1 \%$ chicken embryo extract (Seralab, CE650-DL), and 1\% penicillin/streptomycin (Euroclone, ECB 3001) for 48 or 96 hours, changing the medium only after 72 hours. Myofibers were collected in 2-mL tubes precoated with $10 \%$ FBS/PBS and fixed 15 minutes with $4 \%$ PFA followed by 3 washes in PBS. Permeabilization was performed for 5 minutes with $0.5 \%$ Triton X-100 (MilliporeSigma, 93443)/PBS followed by 2 washes in PBS. Myofibers were incubated 1 hour in blocking solution (10\% FBS/PBS). Primary antibodies were incubated in blocking solution overnight at $4^{\circ} \mathrm{C}$. The day after, myofibers were washed in $0.25 \%$ Tween $20 / \mathrm{PBS}$ twice and incubated 60 minutes with secondary antibodies in blocking solution. Fibers were washed in 0.1\% Tween 20/PBS (PBS-T), incubated 5 minutes with DAPI, briefly washed twice in PBS, and mounted on slides with a drop of Prolong Antifade.

Satellite cells. In order to preserve the integrity of chromatin architecture, MuSC suspensions were fixed in 1\% PFA for 9 minutes and quenched with $125 \mathrm{mM}$ glycine (MilliporeSigma, 8898) before FACS staining and isolation. MuSCs were placed on coverslips precoated with poly-L-lysine (MilliporeSigma, P8920) at a density of 100,000 cells $/ \mathrm{mL}$ for 30 minutes at room temperature. Coverslips were fixed with $4 \% \mathrm{PFA} / \mathrm{PBS}$ for 10 minutes at room temperature.
Then, cells were washed 2 times, 5 minutes each in $0.05 \%$ Triton $\mathrm{X}-100 / \mathrm{PBS}$, permeabilized with 0.5\% Triton X-100/PBS for 10 minutes, and rinsed in PBS. The slides were incubated in $20 \%$ glycerol (MilliporeSigma, G5516)/PBS for at least 60 minutes followed by 4 rounds of freezing and thawing on dry ice (30 seconds), and thawing in $20 \%$ glycerol/PBS at room temperature. Slides were washed 2 times, 5 minutes each in $0.05 \%$ Triton X-100/PBS, once for 5 minutes in the same, incubated in $0.1 \mathrm{M} \mathrm{HCl}$ (MilliporeSigma, H1758) for 15 minutes, and rinsed in PBS. Nonspecific signals were blocked with $1 \%$ $\mathrm{BSA} / \mathrm{PBS}$ for 30 minutes at room temperature. Reaction with primary antibodies Ezh2 diluted 1:100 in blocking solution was performed 12-16 hours at $4^{\circ} \mathrm{C}$; lamin $\mathrm{A} / \mathrm{C}$ diluted 1:200 in blocking solution was performed the day after at room temperature for 2 hours. Secondary antibodies were diluted 1:200 in blocking solution for 1 hour at room temperature. Washes were done in PBS. DNA was counterstained with DAPI, and coverslips were mounted in Prolong Antifade. Primary antibodies are listed in Supplemental Table 2. Secondary antibodies conjugated with the following fluorophores were from Jackson ImmunoResearch: Alexa Fluor 488 (catalog 711-545-152), Cy5 1:300 (catalog 111-115-144), Cy3-streptavidin (catalog 016-160-084), and Alexa Fluor 594 (catalog 711-545-150).

\section{D multicolor DNA FISH}

To produce probes for 3D multicolor DNA FISH, we used the following BAC DNA clones (BACPAC Resources Program, CHORI): CH29101F16 (for Pparg) and CH29-555O5 (for the upstream region of Pparg). One to $3 \mu \mathrm{g}$ of BAC DNA was labeled with dig-dUTP (Roche, 11093088910) (for the upstream region of Pparg) or Cy3-dUTP (Thermo Fisher Scientific, C11401) (for Pparg) through nick translation in $50 \mu \mathrm{L}$ of labeling mix buffer composed of $0.02 \mathrm{mM} \mathrm{C}-\mathrm{G}-\mathrm{A}$ dNTPs (Euroclone, EMR273025, EMR274025, and EMR272025, respectively), $0.01 \mathrm{mM}$ dTTP (Euroclone, EMR275025), $0.01 \mathrm{mM}$ labeled dUTP, 50 mM Tris- $\mathrm{HCl} \mathrm{pH} \mathrm{7.8,} 5 \mathrm{mM} \mathrm{MgCl}_{2}, 10 \mathrm{mM} \beta$-mercaptoethanol, $10 \mathrm{ng} / \mu \mathrm{L}$ BSA, 0.05 to $0.1 \mathrm{U} / \mu \mathrm{L}$ DNA polymerase I (Thermo Fisher Scientific, 18010-017), and 0.004 to $0.001 \mathrm{U} / \mu \mathrm{L}$ Amplification Grade DNase I (MilliporeSigma, D5307) for $30 \mathrm{~min}$ utes to 2 hours at $16^{\circ} \mathrm{C}$, to obtain an average probe size of $50 \mathrm{bp}$. Probes were harvested by ethanol precipitation, resuspended in 10 $\mathrm{mM}$ Tris- $\mathrm{HCl} \mathrm{pH}$ 7.5, and then quantified using a Nanodrop 1000 Spectrophotometer (Thermo Fisher Scientific). For a single experiment, 100 to $300 \mathrm{ng}$ of each probe was precipitated with $3.5 \mu \mathrm{g}$ of mouse Cot-1 DNA (Thermo Fisher Scientific, 18440-016) and $20 \mu \mathrm{g}$ of deoxyribonucleic acid, single stranded from salmon testes (MilliporeSigma, D7656), and then resuspended in $6 \mu \mathrm{L}$ of hybridization solution ( $50 \%$ formamide $\mathrm{pH} 7.0 / 2 \times \mathrm{SSC} / 10 \%$ dextran sulfate). Prefixed satellite cells were plated directly on coverslips precoated with poly-L-lysine and fixed with 4\% PFA in PBS and PBS-T for 10 minutes at room temperature. During the last minute, several drops of $0.5 \%$ Triton $\mathrm{X}-100$ in PBS were added and then cells were washed with $0.01 \%$ Triton $\mathrm{X}-100$ in PBS 3 times for 3 minutes each at room temperature. Cells were first permeabilized with $0.5 \%$ Triton X-100 in PBS for 10 minutes at room temperature. In order to remove RNA, samples were treated with RNase Cocktail Enzyme Mix (Thermo Fisher Scientific, AM2288) for 1 hour at $37^{\circ} \mathrm{C}$. Cells were subjected to other steps of permeabilization with $20 \%$ glycerol in PBS overnight at room temperature, followed by 4 cycles of freezing and thawing in $20 \%$ glycerol in PBS. Permeabilized cells were washed with PBS 
3 times for 10 minutes each at room temperature. Cells were then incubated in $0.1 \mathrm{M} \mathrm{HCl}$ for 5 minutes at room temperature, followed by a rinse with $2 \times$ SSC and then incubated in $50 \%$ formamide in $2 \times$ SSC for at least 30 minutes at room temperature. Slides were equilibrated in $2 \times$ SSC for 2 minutes, washed in PBS for 3 minutes, and then treated with $0.0025 \%$ to $0.0075 \%$ pepsin in $0.01 \mathrm{~N}$ to $0.03 \mathrm{~N} \mathrm{HCl}$ for 2 to 4 minutes at room temperature to eliminate the cytoskeleton. Pepsin was inactivated with $50 \mathrm{mM} \mathrm{MgCl}_{2}$ in PBS twice for 5 minutes each. Nuclei were postfixed with 1\% PFA in PBS for 1 minute, washed with PBS for 5 minutes and with $2 \times$ SSC twice, and then back to $50 \%$ formamide in $2 \times$ SSC for at least 30 minutes at room temperature. Hybridization solution was loaded on a clean microscopic slide, coverslips with nuclei were turned upside down on a drop of hybridization mixture, and sealed with rubber cement. Samples were denatured for 4 minutes at $75^{\circ} \mathrm{C}$ and left to hybridize in a metallic box floating in a $37^{\circ} \mathrm{C}$ water bath overnight. Samples were washed with $2 \times$ SSC 3 times for 5 minutes each at $37^{\circ} \mathrm{C}$ and with $0.1 \times$ SSC 3 times for 5 minutes each at $60^{\circ} \mathrm{C}$, followed by a rinse with $0.2 \%$ Tween 20 in $4 \times$ SSC. Nonspecific binding sites were blocked with blocking solution ( $4 \%$ BSA in $4 \times$ SSC, $0.2 \%$ Tween 20 ) for 20 minutes at $37^{\circ} \mathrm{C}$. Samples were then incubated in the appropriate concentration of streptavidin, DyLight 488-labeled anti-digoxigenin/digoxin (Vector Laboratories, DI-7488) (1:100) diluted in blocking solution for 35 minutes in a dark and wet chamber at $37^{\circ} \mathrm{C}$. Samples were washed with $0.2 \%$ Tween 20 in $4 \times \operatorname{SSC} 3$ times for 3 minutes each at $37^{\circ} \mathrm{C}$, equilibrated in PBS, and postfixed with $2 \%$ formaldehyde in PBS for 10 minutes at room temperature. Finally, the 3D-fixed nuclei were washed with PBS 3 times for 5 minutes each at room temperature, counterstained with $1 \mathrm{ng} / \mu \mathrm{L}$ DAPI in PBS for 10 minutes at room temperature, and washed with PBS 2 times for 5 minutes each at room temperature. After mounting the coverslips, an Eclipse Ti-E (Nikon Instruments) microscope was used to scan the nuclei, with an axial distance of 0.2 - to 0.25 - $\mu \mathrm{m}$ consecutive sections.

\section{Histone extraction and Western blot}

Total proteins were prepared starting from quadriceps muscle. Muscles were homogenized on ice with a Tissue RUptor (Qiagen, 9002755) in $1 \mathrm{~mL}$ of extraction buffer composed of $50 \mathrm{mM}$ Tris- $\mathrm{HCl}, \mathrm{pH} 7.5,150$ $\mathrm{mM} \mathrm{NaCl}, 1 \mathrm{mM}$ EDTA pH 8.0, 0.1\% SDS, 1\% NP-40 (MilliporeSigma, 74385), $0.5 \%$ sodium deoxycholate (MilliporeSigma, D6750), $1 \times$ protease inhibitors (Roche, 04693132001), 2 mM PMSF (MilliporeSigma, 93482), $1 \mathrm{mM} \mathrm{NaF}$ (MilliporeSigma, s7920), and $1 \mathrm{mM} \mathrm{Na}_{3} \mathrm{VO}_{4}$. After 30 minutes in ice, 3 pulses of 10 -second sonication at $30 \%$ amplitude were performed with a Branson digital sonifier 250 to promote dissociation of protein from chromatin and solubilization. Extracts were analyzed by SDS-PAGE using $8 \%$ to $10 \%$ gels (29:1 acrylamide solution; MilliporeSigma, 01708). For histone extractions, approximately $2 \times 10^{6}$ freshly isolated MuSCs were collected and resuspended in 15 $\mu \mathrm{L}$ of $0.2 \mathrm{~N} \mathrm{HCl}$ with $1 \times$ protease inhibitors, $2 \mathrm{mM}$ PMSF, $1 \mathrm{mM} \mathrm{NaF}$, and $1 \mathrm{mM} \mathrm{Na}_{3} \mathrm{VO}_{4}$, and incubated overnight at $4^{\circ} \mathrm{C}$ with constant rocking. The supernatant was run in $4 \%$ to $12 \%$ Bis-Tris acrylamide gels (Thermo Fisher Scientific, NW04125). Primary antibodies are listed in Supplemental Table 2.

\section{RNA-seq}

Total RNA from freshly isolated satellite cells from 3 mice for each genotype was extracted in TRI Reagent (MilliporeSigma, T9424) following the manufacturer's guidelines. Libraries were prepared using the Illumina TruSeq Stranded Total RNA with Ribo-Zero GOLD kit and were quality controlled with an Agilent Bioanalyzer at the Sequencing Facility of the Institute of Applied Genomics (IGA, Udine, Italy). Reads (125 bp) were produced using an Illumina HiSeq 2500 instrument in paired-end mode to reach a sequencing depth of approximately 30 million reads for each sample. RT-PCR was performed on total RNA extracted from individual mice. Primers sequences were the following: Gapdh, 5'-GTATGTCGTGGAGTCTACTGG-3' and 5'-TCGTGGTTCACACCCATCAC-3'; p16, 5'-GTGTGCATGACGTGCGGG-3' and 5'-CAGTTCGAATCTGCACCGTAG-3'; p19, 5'-GCTCTGGCTTTCGTGAACATG-3' and 5'-TCGAATCTGCACCGTAGTTGAG-3'; p21, 5'-ACGGAGGCAGACCAGCCT-3' and 5'-ACACAGAGTGAGGGCTAAGG-3'.

\section{ChIP-seq}

For ChIP analysis, satellite cells from pools of 6 to 9 mice were used and prefixed as described above in the Immunofluorescence section. Isolated satellite cells were resuspended in SDS buffer $(100 \mathrm{mM}$ $\mathrm{NaCl}, 50 \mathrm{mM}$ Tris- $\mathrm{HCl} \mathrm{pH}$ 8.1, 5 mM EDTA, $0.2 \% \mathrm{NaN}_{3}, 0.5 \%$ SDS) and stored at $-80^{\circ} \mathrm{C}\left(100 \mu \mathrm{L} / 10^{6}\right.$ cells $)$. Fixed cells $\left(2 \times 10^{6}\right.$ to $2.5 \times$ $10^{6}$ per experiment) were thawed on ice, resuspended in fresh SDS buffer, and incubated at $4^{\circ} \mathrm{C}$ with mild agitation for 3 hours, passing them through a $0.50 \times 16 \mathrm{~mm}$ syringe needle every hour. The solution was then adjusted to IP buffer composition (100 mM Tris $\mathrm{pH}$ 8.6, 0.3\% SDS, 1.7\% Triton X-100, 5 mM EDTA) and cells were sonicated with a Branson Digital Sonifier to obtain 200-bp fragments. For spike-in experiments, $5 \%$ sheared Drosophila chromatin was added to each sample. Two percent of the total volume from each sample was taken as input chromatin. The remaining fragmented chromatin was incubated with $1 \mathrm{mM}$ PMSF and $4 \mu \mathrm{g}$ of the antibody of interest on a rotating wheel at $4^{\circ} \mathrm{C}$ overnight. Primary antibodies used were H3K27me3 (Millipore, 07-449) and H3K4me3 (Millipore, 07-473). The next day, protein G beads (Life Technologies, 1004D) were added $(80 \mu \mathrm{L})$ and samples were incubated for an additional 2 hours on the rotating wheel at $4^{\circ} \mathrm{C}$. The beads were washed with lowsalt solution (150 mM NaCl, 20 mM Tris- $\mathrm{HCl}$ pH 8.0, 2 mM EDTA, $0.1 \%$ SDS, $1 \%$ Triton X-100), high-salt solution (500 mM NaCl, 20 mM Tris-HCl pH 8.0, 2 mM EDTA, 0.1\% SDS, 1\% Triton X-100), and low-salt solution and then $\mathrm{TE} \mathrm{NaCl}(50 \mathrm{mM} \mathrm{NaCl}, 10 \mathrm{mM}$ Tris- $\mathrm{HCl}$ pH 8.0, $1 \mathrm{mM}$ EDTA). Crosslinking was reversed by incubating the beads at $65^{\circ} \mathrm{C}$ overnight in elution buffer $(50 \mathrm{mM}$ Tris- $\mathrm{HCl} \mathrm{pH} \mathrm{8.0,}$ $10 \mathrm{mM}$ EDTA, 1\% SDS). Input chromatin was also de-crosslinked in elution buffer overnight at $65^{\circ} \mathrm{C}$. The next day, all samples were diluted with 1 volume of TE 10:1, treated with $0.2 \mu \mathrm{g} / \mathrm{mL}$ RNase A (MilliporeSigma, R6513) for 2 hours at $37^{\circ} \mathrm{C}$, and then with $0.2 \mu \mathrm{g} / \mathrm{mL}$ Proteinase K (MilliporeSigma, P2308) for 2 hours at $55^{\circ} \mathrm{C}$. DNA was isolated through standard phenol/chloroform extraction, followed by precipitation and resuspension in $31 \mu \mathrm{L}$ of $10 \mathrm{mM}$ Tris- $\mathrm{HCl} \mathrm{pH}$ 8.0. Before library construction, ChIPs were validated using the following primers: Hoxd9, 5'-GGATAATCGCCTAGGTGTGACTT-3' and $5{ }^{\prime}$-CATCTCTTCTTGCCTCTCTGGG-3'; Pax7 promoter, 5'-GCGACCCCCTGAGGAAAA-3' and 5'-CGAAAAGAAGTCTCCAACGAGTATT-3'. Libraries for IP and reference input DNA were created for each sample using the Biomek FX (Beckman Coulter), and then qualitatively and quantitatively checked using the Agilent High Sensitivity DNA Kit (Agilent Technologies, 5067-4627) on a 
Bioanalyzer 2100 (Agilent Technologies). Libraries with distinct adapter indexes were multiplexed and, after cluster generation on FlowCell, were sequenced for 50 bases in the single-read mode on a HiSeq 2000 sequencer at the IEO Genomic Unit in Milan.

\section{Real-time PCR analysis}

Total RNA was extracted from satellite cells using a Maxwell RSC miRNA tissue kit (Promega, AS1460), while total RNA from muscle tissues was extracted using TRI Reagent (MilliporeSigma, T9424). cDNA was synthesized from $1 \mu \mathrm{g}$ of RNA from each sample using a QuantiTect reverse transcription kit (Qiagen, 205313) and amplified in the presence of $8 \mu \mathrm{L}$ of SYBR Select Master Mix (Thermo Fisher Scientific, 4472908). Expression was calculated by normalizing to Gapdh and relative to the average of the WT control samples. Primer sequences used for transcriptional analyses were the following: mouse mRTP21, 5'-ACGGAGGCAGACCAGCCT-3' and 5'-ACACAGAGTGAGGGCTAAGG-3'; mrtezh2, 5'-CAAATACATGTGCAGCTTTCTG-3' and 5'-ATGCCTATCCTGTGGTCACC-3'; mrtpparg, 5'-TTGCTGAACGTGAAGCCCATCGAG-3' and 5'-GTCCTTGTAGATCTCCTGGAGCAG-3'; mrtp16, 5'-GTGTGCATGACGTGCGGG-3' and 5'-CAGTTCGAATCTGCACCGTAG-3'; mrtp19, 5'-GCTCTGGCTTTCGTGAACATG-3' and 5'-TCGAATCTGCACCGTAGTTGAG-3'; mrtgapdh, 5'-GTATGTCGTGGAGTCTACTGG-3' and 5'-TCGTGGTTCACACCCATCAC-3'.

\section{Bioinformatics and image analysis}

See Supplemental Methods.

\section{Data availability}

The data sets generated during the current study are available in the NCBI's Gene Expression Omnibus (GEO) repository with accession number GSE123725.

\section{Statistics}

All the data were analyzed using GraphPad Prism 6. The sample size ( $n$ ) is described for each experiment in the respective figure legend. Multiple comparisons between 3 or more groups were made using 1-way or 2-way ANOVA with significance set as ${ }^{*} P<0.05 ;{ }^{* *} P<0.01 ;{ }^{* *} P<0.001$.

\section{Study approval}

All the experimental procedures were performed under the ethical approval of the Italian Ministry of Health and the Institutional Animal Care and Use Committee (authorization no. 83/2019-PR). The animals were maintained in an authorized facility at San Raffaele Hospital, Milan (authorization no. 127/2012-A) and Santa Lucia Foundation, Rome (authorization no. 9/2006-A).

\section{Author contributions}

$\mathrm{CL}$ conceived the project and designed experiments. $\mathrm{AB}$ and $\mathrm{CM}$ performed experiments and analyzed data. FL and DP performed and interpreted ChIP-seq experiments. SV performed bioinformatic analysis. GP, VR, RR, and $\mathrm{CB}$ performed experiments in mice. $\mathrm{AB}$, $\mathrm{AC}$, and $\mathrm{BB}$ performed and interpreted FISH experiments. LA, FG, and GO quantified immunofluorescence and FISH images. MDB performed FACS. FF and CP analyzed ChIP-seq data. CL supervised the project and analyzed data. $\mathrm{CL}, \mathrm{AB}$, and $\mathrm{CM}$ wrote the manuscript. All authors edited and approved the manuscript.

\section{Acknowledgments}

We thank Maria Vivo, Gisèle Bonne, Federica Marasca, Pierluigi Manti, Davide Gabellini, Lorenzo Puri, Daniela Palacios, Giovanna Lattanzi, the Italian network of laminopathies, and members of the laboratory for stimulating discussions and constructive criticisms. We are grateful to Chiara Cordiglieri and INGM Imaging Facility for assistance during image acquisition and to Mariacristina Crosti, Monica Moro, and the INGM FACS Facility for assistance in cell sorting. This work was supported by grants from the Italian Minister of Health no. GR-2013-02355413 to CL, My First AIRC Grant (MFAG) no. 18535 to CL, AFM-Telethon no. 21030 to CL and FF, and Cariplo 2017-0649 to CL and FF.

Address correspondence to: Lanzuolo Chiara. Via Fratelli Cervi, 93, 20090 Segrate (MI), Italy. Phone: 39.02.00660358; Email: lanzuolo@ingm.org. MC's present address is: CNR Institute of Molecular Biology and Pathology (IBPM), Rome, Italy. VS's present address is: San Raffaele Telethon Institute for Gene Therapy (SR-Tiget), IRCCS San Raffaele Scientific Institute, Milan, Italy.
1. Shimi $T$, et al. Structural organization of nuclear lamins A, C, B1, and B2 revealed by superresolution microscopy. Mol Biol Cell. 2015;26(22):4075-4086.

2. Turgay Y, et al. The molecular architecture of lamins in somatic cells. Nature. 2017;543(7644):261-264.

3. van Steensel B, Belmont AS. Lamina-associated domains: links with chromosome architecture, heterochromatin, and gene repression. Cell. 2017;169(5):780-791.

4. Zaremba-Czogalla M, Dubińska-Magiera M, Rzepecki R. Laminopathies: the molecular background of the disease and the prospects for its treatment. Cell Mol Biol Lett. 2011;16(1):114-148.

5. Kolb T, Maass K, Hergt M, Aebi U, Herrmann H. Lamin A and lamin C form homodimers and coexist in higher complex forms both in the nucleoplasmic fraction and in the lamina of cultured human cells. Nucleus. 2011;2(5):425-433.

6. Serebryannyy L, Misteli T. Protein sequestration at the nuclear periphery as a potential regulatory mechanism in premature aging. J Cell Biol. 2018;217(1):21-37.

7. Dubinska-Magiera M, Zaremba-Czogalla M, Rzepecki R. Muscle development, regeneration and laminopathies: how lamins or lamina-associated proteins can contribute to muscle development, regeneration and disease. Cell Mol Life Sci. 2013;70(15):2713-2741.

8. Schuettengruber B, Bourbon HM, Di Croce L, Cavalli G. Genome regulation by polycomb and trithorax: 70 years and counting. Cell. 2017;171(1):34-57.

9. Briand N, Collas P. Laminopathy-causing lamin A mutations reconfigure lamina-associated domains and local spatial chromatin conformation. Nucleus. 2018;9(1):216-226.

10. Cesarini E, et al. Lamin A/C sustains PcG protein architecture, maintaining transcriptional repression at target genes. JCell Biol. 2015;211(3):533-551.
11. Marullo F, Cesarini E, Antonelli L, Gregoretti F, Oliva G, Lanzuolo C. Nucleoplasmic lamin A/C and polycomb group of proteins: An evolutionarily conserved interplay. Nucleus. 2016;7(2):103-111.

12. Oldenburg A, et al. A lipodystrophy-causing lamin A mutant alters conformation and epigenetic regulation of the anti-adipogenic MIR335 locus. J Cell Biol. 2017;216(9):2731-2743.

13. Salvarani N, et al. The K219T-lamin mutation induces conduction defects through epigenetic inhibition of SCN5A in human cardiac laminopathy. Nat Commun. 2019;10(1):2267.

14. Zheng $X$, et al. Lamins organize the global three-dimensional genome from the nuclear periphery. Mol Cell. 2018;71(5):802-815.e7.

15. Chiacchiera F, et al. Polycomb complex PRC1 preserves intestinal stem cell identity by sustaining Wnt/ $\beta$-catenin transcriptional activity. Cell Stem Cell. 2016;18(1):91-103.

16. Lapthanasupkul $P$, et al. Ring1a/b polycomb 
proteins regulate the mesenchymal stem cell niche in continuously growing incisors. Dev Biol. 2012;367(2):140-153.

17. Bernstein BE, et al. A bivalent chromatin structure marks key developmental genes in embryonic stem cells. Cell. 2006;125(2):315-326.

18. Morata G, Herrera SC. Cell reprogramming during regeneration in Drosophila: transgression of compartment boundaries. Curr Opin Genet Dev. 2016;40:11-16.

19. Lu TT, et al. The polycomb-dependent epigenome controls $\beta$ cell dysfunction, dedifferentiation, and diabetes. Cell Metab. 2018;27(6):1294-1308.e7.

20. Cohen TV, et al. Defective skeletal muscle growth in lamin A/C-deficient mice is rescued by loss of Lap2 $\alpha$. Hum Mol Genet. 2013;22(14):2852-2869.

21. Sullivan T, et al. Loss of A-type lamin expression compromises nuclear envelope integrity leading to muscular dystrophy. JCell Biol. 1999;147(5):913-920.

22. Gregoretti F, Cesarini E, Lanzuolo C, Oliva G, Antonelli L. An automatic segmentation method combining an active contour model and a classification technique for detecting polycomb-group proteinsin high-throughput microscopy images. Methods Mol Biol. 2016;1480:181-197.

23. Boonsanay V, et al. Regulation of skeletal muscle stem cell quiescence by Suv4-20h1-dependent facultative heterochromatin formation. Cell Stem Cell. 2016;18(2):229-242.

24. Juan AH, et al. Polycomb EZH2 controls selfrenewal and safeguards the transcriptional identity of skeletal muscle stem cells. Genes Dev. 2011;25(8):789-794.

25. Liu L, et al. Chromatin modifications as determinants of muscle stem cell quiescence and chronological aging. Cell Rep. 2013;4(1):189-204.

26. Orlando DA, et al. Quantitative ChIP-Seq normalization reveals global modulation of the epigenome. Cell Rep. 2014;9(3):1163-1170.

27. Minoux M, et al. Gene bivalency at polycomb domains regulates cranial neural crest positional identity. Science. 2017;355(6332):eaal2913.

28. Bernstein E, Duncan EM, Masui O, Gil J, Heard E, Allis $\mathrm{CD}$. Mouse polycomb proteins bind differentially to methylated histone $\mathrm{H} 3$ and RNA and are enriched in facultative heterochromatin. Mol Cell Biol. 2006;26(7):2560-2569.

29. Barak $Y$, et al. PPAR gamma is required for placental, cardiac, and adipose tissue development. Mol Cell.1999;4(4):585-595.

30. Rosen ED, et al. PPAR gamma is required for the differentiation of adipose tissue in vivo and in vitro. Mol Cell. 1999;4(4):611-617.
31. Tong J, Li W, Vidal C, Yeo LS, Fatkin D, Duque G. Lamin A/C deficiency is associated with fat infiltration of muscle and bone. Mech Ageing Dev. 2011;132(11-12):552-559.

32. Bantignies F, et al. Polycomb-dependent regulatory contacts between distant Hox loci in Drosophila. Cell. 2011;144(2):214-226.

33. Lanzuolo C, Roure V, Dekker J, Bantignies F, Orlando V. Polycomb response elements mediate the formation of chromosome higher-order structures in the bithorax complex. Nat Cell Biol. 2007;9(10):1167-1174

34. Dixon JR, et al. Topological domains in mammalian genomes identified by analysis of chromatin interactions. Nature. 2012;485(7398):376-380.

35. Bonev B, et al. Multiscale 3D genome rewiring during mouse neural development. Cell. 2017;171(3):557-572.e24.

36. Wang Y, et al. The 3D Genome Browser: a webbased browser for visualizing 3D genome organization and long-range chromatin interactions. Genome Biol. 2018;19(1):151.

37. Chakkalakal JV, Jones KM, Basson MA, Brack AS The aged niche disrupts muscle stem cell quiescence. Nature. 2012;490(7420):355-360.

38. Gonzalo S, Kreienkamp R, Askjaer P. HutchinsonGilford progeria syndrome: A premature aging disease caused by LMNA gene mutations. Ageing Res Rev. 2017;33:18-29.

39. Jacobs JJ, Kieboom K, Marino S, DePinho RA, van Lohuizen $\mathrm{M}$. The oncogene and polycomb-group gene bmi-1 regulates cell proliferation and senescence through the ink4a locus. Nature. 1999;397(6715):164-168.

40. Chang NC, Chevalier FP, Rudnicki MA. Satellite cells in muscular dystrophy - lost in polarity. Trends Mol Med. 2016;22(6):479-496.

41. Bernet JD, Doles JD, Hall JK, Kelly Tanaka K, Carter TA, Olwin BB. p38 MAPK signaling underlies a cell-autonomous loss of stem cell selfrenewal in skeletal muscle of aged mice. $\mathrm{Nat}$ Med. 2014;20(3):265-271.

42. Cosgrove $\mathrm{BD}$, et al. Rejuvenation of the muscle stem cell population restores strength to injured aged muscles. Nat Med. 2014;20(3):255-264.

43. Kuang S, Kuroda K, Le Grand F, Rudnicki MA. Asymmetric self-renewal and commitment of satellite stem cells in muscle. Cell. 2007;129(5):999-1010.

44. Sousa-Victor P, et al. Geriatric muscle stem cells switch reversible quiescence into senescence. Nature. 2014;506(7488):316-321.

45. Béguelin W, et al. EZH2 enables germinal centre formation through epigenetic silencing of CDKN1A and an Rb-E2F1 feedback loop. Nat Commun. 2017;8(1):877.

46. Yosef R, et al. p21 maintains senescent cell viability under persistent DNA damage response by restraining JNK and caspase signaling. $Е M B O$ J. 2017;36(15):2280-2295.

47. Kandert S, Wehnert M, Müller CR, Buendia B, Dabauvalle MC. Impaired nuclear functions lead to increased senescence and inefficient differentiation in human myoblasts with a dominant p.R545C mutation in the LMNA gene. Eur JCell Biol. 2009;88(10):593-608.

48. Quelle DE, et al. Cloning and characterization of murine p16INK $4 \mathrm{a}$ and p15INK $4 \mathrm{~b}$ genes. Oncogene. 1995;11(4):635-645.

49. Ito T, Teo YV, Evans SA, Neretti N, Sedivy JM. Regulation of cellular senescence by polycomb chromatin modifiers through distinct DNA Damage- and histone methylation-dependent pathways. Cell Rep. 2018;22(13):3480-3492.

50. Serrano M, Lee H, Chin L, Cordon-Cardo C, Beach D, DePinho RA. Role of the INK4a locus in tumor suppression and cell mortality. Cell. 1996;85(1):27-37.

51. Gnocchi VF, Ellis JA, Zammit PS. Does satellite cell dysfunction contribute to disease progression in Emery-Dreifuss muscular dystrophy? Biochem Soc Trans. 2008;36(pt 6):1344-1349.

52. Robinson DCL, Dilworth FJ. Epigenetic regulation of adult myogenesis. Curr Top Dev Biol. 2018;126:235-284.

53. Tosic M, et al. Lsd1 regulates skeletal muscle regeneration and directs the fate of satellite cells. Nat Commun. 2018;9(1):366.

54. Frock RL, Kudlow BA, Evans AM, Jameson SA, Hauschka SD, Kennedy BK. Lamin A/C and emerin are critical for skeletal muscle satellite cell differentiation. Genes Dev. 2006;20(4):486-500.

55. Solovei I, et al. LBR and lamin A/C sequentially tether peripheral heterochromatin and inversely regulate differentiation. Cell. 2013;152(3):584-598.

56. Melcon G, et al. Loss of emerin at the nuclear envelope disrupts the Rb1/E2F and MyoD pathways during muscle regeneration. Hum Mol Genet. 2006;15(4):637-651.

57 . Kundu S, et al. Polycomb repressive complex 1 generates discrete compacted domains that change during differentiation. Mol Cell. 2017;65(3):432-446.e5.

58. Sousa-Victor P, Perdiguero E, Muñoz-Cánoves P. Geroconversion of aged muscle stem cells under regenerative pressure. Cell Cycle. 2014;13(20):3183-3190. 